\title{
Modeling of Tsunami Generation and Propagation by a Spreading Curvilinear Seismic Faulting in Linearized Shallow-Water Wave Theory
}

\author{
Hossam S. Hassan ${ }^{1}$, Khaled T. Ramadan ${ }^{1}$, Sarwat N. Hanna ${ }^{2}$ \\ ${ }^{1}$ Department of Basic and Applied Science, College of Engineering \& Technology, Arab Academy for Science, \\ Technology and Maritime Transport, Alexandria, Egypt \\ ${ }^{2}$ Department of Engineering Mathematics and Physics, Faculty of Engineering, Alexandria University, \\ Alexandria, Egypt \\ E-mail:hossams@aast.edu
}

Received March 8, 2010; revised April 14, 2010; accepted April 23, 2010

\begin{abstract}
The processes of tsunami evolution during its generation in search for possible amplification mechanisms resulting from unilateral spreading of the sea floor uplift is investigated. We study the nature of the tsunami build up and propagation during and after realistic curvilinear source models represented by a slowly uplift faulting and a spreading slip-fault model. The models are used to study the tsunami amplitude amplification as a function of the spreading velocity and rise time. Tsunami waveforms within the frame of the linearized shallow water theory for constant water depth are analyzed analytically by transform methods (Laplace in time and Fourier in space) for the movable source models. We analyzed the normalized peak amplitude as a function of the propagated uplift length, width and the average depth of the ocean along the propagation path.
\end{abstract}

Keywords: Tsunami Modeling, Shallow Water Theory, Water Wave, Bottom Topography, Laplace and Fourier Transforms

\section{Introduction}

Waves at the surface of a liquid can be generated by various mechanisms such as, wind blowing on the free surface, wave maker, moving disturbance on the bottom or the surface, or even inside the liquid, fall of an object into the liquid, liquid inside a moving container, etc. The generation of tsunamis by a seafloor deformation is an example for the case where the waves are created by a given motion of the bottom. There are different natural phenomena that can lead to a tsunami. For example, one can mention submarine slumps, slides, volcanic explosions, earthquakes, etc.

The sea bottom deformation following an underwater earthquake is a complex phenomenon. This is why, for theoretical or experimental studies, researchers have often used simplified bottom motions such as the vertical motion of a box. Most investigations of tsunami generation and propagation used developed integral solution (in space and time) for an arbitrary bed displacement based on a linearized description of wave motion in either a two or three-dimensional fluid domain of uniform depth.
The complexity of the integral solutions developed from the linear theory even for the simplest model of bed deformation prevented many authors from determining detailed wave behaviour, especially near the source region. However, we construct three-dimensional curvilinear source models involved in the transform methods that may generate a tsunami near the source region.

Many authors have used different analytical solutions and numerical computations to determine the general wave pattern near and far from the source region for a variety of bed motions in a two or three-dimensional fluid domain. Ben-Menahem and Rosenman [1] calculated the two-dimensional radiation pattern from a moving source using linear theory. Tuck and Hwang [2] solved the linear long-wave equation in the presence of a moving bottom and a uniformly sloping beach. According to Synolakis and Bernard [3], Houston and Garcia [4] were the first to use more geophysical realistic initial conditions. Tinti and Bortolucci [5] investigated analytically the generation of tsunamis by submarine slides. They specialized the general solution of the 1D Cauchy linear problems for long water waves to deal with rigid 
body to explore the characteristics of the generated waves. They studied the body motion in terms of Froude number, wave pattern, wave amplitude and wave energy, see also [6-13].

Since the general features of the waves obtained by these authors for both the near and far fields are based on a linear theory, their applicability is limited to bed deformations and the range of propagation for which nonlinear effects remain small.

Several works done are taken into account the nonlinear effect in the wave motion for modeling the tsunami waves. Kervella et al. [14] perform a comparison between three-dimensional linear and nonlinear tsunami generation models. They observed very good agreement from the superposition of the wave profiles computed with the linear and fully nonlinear models. Second, they found that the nonlinear shallow water model was not sufficient to model some of the waves generated by a moving bottom because of the presence of frequency dispersion, hence the suggested that for most events the linear theory is sufficient. Villeneuve [15] derived model equations which combine the linear effect of frequency dispersion and the nonlinear effect of amplitude dispersion including the effects of a moving bed. Liu and Liggett [16] performed comparisons between linear and nonlinear water waves where their study was restricted to simple bottom deformations, namely the generation of transient waves by an upthrust of a rectangular block. Bona et al. [17] assessed how well a model equation with weak nonlinearity and dispersion describes the propagation of surface water waves generated at one end of a long channel. In their experiments, they found that the inclusion of a dissipative term was more important than the inclusion of nonlinearity, although the inclusion of nonlinearity was undoubtedly beneficial in describing the observations. Abo Dina et al. [18] have adopted a nonlinear theory and constructed a numerical model of tsunami generation and propagation which permits a variable bed displacement with an arbitrary water depth to be included in the model. In this model, he considered nonlinearities and omitted the linear effects of frequency dispersion; hence, no insight into the possible importance of the interaction of nonlinear and linear effects in the far field was possible, see also [19,20]. All the previous studies mention above neglected the details of wave generation in fluid during the source time. One of the reasons is that it is commonly assumed that the source details are not important.

The transient wave generation due to the coupling between the seafloor motion and the free surface has been considered by a few authors only. There are some specific cases where the time scale of the bottom deformation and the horizontal extent of the bottom deformation may become an important factor. Some studies have already been performed to understand wave formation due to different prescribed bottom motions by introduc- ing either some type of rise time or some type of rupture velocity. For example, Todorovska et al. [21] studied the generation of waves by a slowly spreading uplift of the bottom in linearized shallow-water wave theory and where able to explain some observations. They studied the tsunami amplitude amplification as a function of the model parameters. They found the effects of the spreading of the ocean floor deformation (faulting, submarine slides or slumps) on the amplitudes and periods of the generated tsunamis are largest when the spreading velocity of uplift and the tsunami velocity are comparable. Trifunac et al. [22] mentioned the source parameters for submarine slides and earthquakes including source duration, displacement amplitude, areas and volumes of selected past earthquakes that have or may have generated a tsunami. They contributed the nature of tsunami sources to create tsunami waveforms in the near field and provided a starting point for their elementary mathematical model. Todorovska et al. [23] investigated tsunami generation by a slowly spreading uplift of the sea floor in the near field considering the effects of the source finiteness and directivity. They described mathematically various two-dimensional kinematic models of submarine slumps and slides as combinations of spreading constant or slopping uplift functions. There results show that for given constant water depth, the peak amplitude depends on the ratio of the spreading velocity of the sea floor to the long wavelength tsunami velocity, see also their works [24,25]. Hammack [26] generated waves experimentally by raising or lowering a box at one end of a channel. He considered two types of time histories: an exponential and a half-sine bed movement. Dutykh and Dias [27] generated waves theoretically by multiplying the static deformations caused by slip along a fault by various time laws: instantaneous, exponential, trigonometric and linear. Haskell [28] was one of the first authors, who take into account the rupture velocity. In fact he considered both rise time $\mathrm{T}$ and rupture velocity $V$.

All the previous approaches done in [21-28] computed tsunami waveforms using linearized shallow water theory and transform methods of solution. We follow the same approach but with a more realistic and more complex source models. This approach is restricted to the water region where the incompressible Euler equations for potential flow can be linearized. In this paper we investigate the tsunami wave in the near and far field using the transform methods (Laplace in time and Fourier in space). We construct mathematically a reasonable curvilinear tsunami source based on available geological, seismological, and tsunami elevation. This model resembles the initial source predicted according to the initial disturbance recorded in $[29,30]$. We discuss aspects of tsunami generation that should be considered in developing these models, as well as the propagation wave after the formation of the source models have been completed. 
We study the fluid wave motion above finite sources, with irregular fault shapes and with variable distribution of the ocean floor uplift, for variable spreading velocities. Here we aim to demonstrate the large scale tsunami generation features computed during the formation of the tsunami source for different ratios between the velocity of the source propagation and the tsunami speed, as well as the overall propagation following the source. Comparison between our results and others obtained for the tsunami model in the near-field is done. According to the results and the numerical estimation, we analyze the normalized peak amplitude as a function of the characteristics size of the source model and the water depth.

\section{Mathematical Formulation of the Problem}

Consider a three dimensional fluid domain $\Omega$ as shown in Figure 1. It is supposed to represent the ocean above the fault area. It bounded above by the free surface of the ocean $\mathrm{z}=\eta(\mathrm{x}, \mathrm{y}, \mathrm{t})$ and below by the rigid ocean floor $\mathrm{z}$ $=-\mathrm{h}(\mathrm{x}, \mathrm{y})+\zeta(\mathrm{x}, \mathrm{y}, \mathrm{t})$, where $\eta(\mathrm{x}, \mathrm{y}, \mathrm{t})$ is the free surface elevation, $\mathrm{h}(\mathrm{x}, \mathrm{y})$ is the water depth and $\zeta(\mathrm{x}, \mathrm{y}, \mathrm{t})$ is the sea floor displacement function. The domain $\Omega$ is unbounded in the horizontal directions $\mathrm{x}$ and $\mathrm{y}$, and can be written as $\Omega=\mathrm{R}^{2} \times[-\mathrm{h}(\mathrm{x}, \mathrm{y})+\zeta(\mathrm{x}, \mathrm{y}, \mathrm{t}), \eta(\mathrm{x}, \mathrm{y}, \mathrm{t})]$. For simplicity, $\mathrm{h}(\mathrm{x}, \mathrm{y})$ is assumed to be a constant. Before the earthquake, the fluid is assumed to be at rest, thus the free surface and the solid boundary are defined by $z=0$ and $\mathrm{z}=-\mathrm{h}$, respectively. Mathematically, these conditions can be written in the form of initial conditions: $\eta(\mathrm{x}$, $\mathrm{y}, 0)=\zeta(\mathrm{x}, \mathrm{y}, 0)=0$. At time $\mathrm{t}>0$ the bottom boundary moves in a prescribed manner which is given by $\mathrm{z}=-\mathrm{h}+$ $\zeta(\mathrm{x}, \mathrm{y}, \mathrm{t})$. The deformation of the sea bottom is assumed to have all the necessary properties needed to compute its Fourier transform in $\mathrm{x}, \mathrm{y}$ and its Laplace transform in $\mathrm{t}$. The resulting deformation of the free surface $\mathrm{z}=\eta(\mathrm{x}, \mathrm{y}, \mathrm{t})$ is to be found as part of the solution. It is assumed that the fluid is incompressible and the flow is irrotational. The former implies the existence of a velocity potential $\phi(\mathrm{x}, \mathrm{y}, \mathrm{z}, \mathrm{t})$ which fully describes the flow and the physical process. By definition of $\phi$, the fluid velocity vector can be expressed as $\overrightarrow{\mathrm{q}}=\nabla \phi$. Thus, the potential

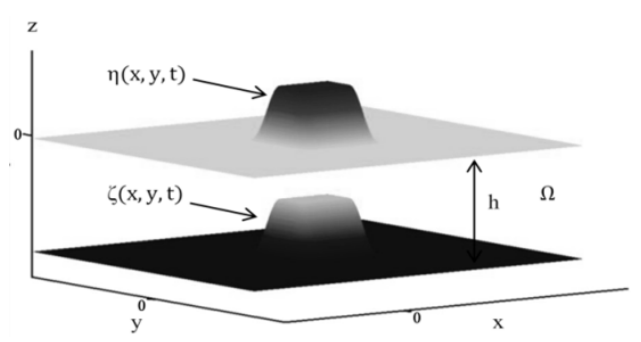

Figure 1. Definition of the fluid domain and coordinate system for a very rapid movement of the assumed source model. flow $\phi(\mathrm{x}, \mathrm{y}, \mathrm{z}, \mathrm{t})$ must satisfy the Laplace's equation

$$
\nabla^{2} \phi(\mathrm{x}, \mathrm{y}, \mathrm{z}, \mathrm{t})=0 \text { where }(\mathrm{x}, \mathrm{y}, \mathrm{z}) \in \Omega
$$

The potential $\phi(x, y, z, t)$ must satisfy the following kinematic and dynamic boundary conditions on the free surface and the solid boundary, respectively:

$$
\begin{array}{r}
\phi_{\mathrm{z}}=\eta_{\mathrm{t}}+\phi_{\mathrm{x}} \eta_{\mathrm{x}}+\phi_{\mathrm{y}} \phi_{\mathrm{y}} \quad \text { on } \mathrm{z}=\eta(\mathrm{x}, \mathrm{y}, \mathrm{t}), \\
\phi_{\mathrm{z}}=\zeta_{\mathrm{t}}+\phi_{\mathrm{x}} \zeta_{\mathrm{x}}+\phi_{\mathrm{y}} \zeta_{\mathrm{y}} \quad \text { on } \mathrm{z}=-\mathrm{h}+\zeta(\mathrm{x}, \mathrm{y}, \mathrm{t}),
\end{array}
$$

and

$$
\phi_{\mathrm{t}}+\frac{1}{2}(\nabla \phi)^{2}+g \eta=0 \quad \text { on } \mathrm{z}=\eta(\mathrm{x}, \mathrm{y}, \mathrm{t}),
$$

where $\mathrm{g}$ is the acceleration due to gravity. As described above, the initial conditions are given by

$$
\phi(\mathrm{x}, \mathrm{y}, \mathrm{z}, 0)=\eta(\mathrm{x}, \mathrm{y}, 0)=\zeta(\mathrm{x}, \mathrm{y}, 0)=0 .
$$

\subsection{Linear Shallow Water Theory}

Various approximations can be considered for the full water-wave equations. One is the system of Boussinesq equations that retains nonlinearity and dispersion up to a certain order. Boussinesq model is used to study transient varying bottom problems. Fuhrman et al. [31] and Zhao et al. [32] presented a developed numerical model based on the highly accurate Boussinesq-type formulation subjected to exact expressions for the kinematic and dynamic free surface conditions. Their results show that the model was capable of treating the full life cycle of tsunami evolution, from the initial generation of bottom movements, to the subsequent propagation, and through the final the run-up process. Reasonable computational efficiency has been demonstrated in their work, which made the model attractive for practical coastal engineering studies, where high dispersive and nonlinear accuracy is sought. Another one is the system of nonlinear shallow-water equations that retains nonlinearity but no dispersion. Solving this problem is a difficult task due to the nonlinearities and the a priori unknown free surface. The simplest one is the system of linear shallow-water equations. The concept of shallow water is based on the smallness of the ratio between water depth and wave length. In the case of tsunamis propagating on the surface of deep oceans, one can consider that shallow-water theory is appropriate because the water depth (typically several kilometers) is much smaller than the wave length (typically several hundred kilometers), which is reasonable and usually true for most tsunamis triggered by submarine earthquakes, slumps and slides [26,27]. Hence, the problem can be linearized by neglecting the nonlinear terms in the boundary conditions (2)-(4) and if the boundary conditions are applied on the nondeformed instead of the deformed boundary surfaces (i.e., on $\mathrm{z}=-\mathrm{h}$ and $\mathrm{z}=0$ instead of $\mathrm{z}=-\mathrm{h}+\zeta(\mathrm{x}, \mathrm{y}, \mathrm{t})$ and $\mathrm{z}=\eta(\mathrm{x}, \mathrm{y}, \mathrm{t}))$.

The linearized problem in dimensional variables can be written as 
$\nabla^{2} \phi(\mathrm{x}, \mathrm{y}, \mathrm{z}, \mathrm{t})=0$ where $(\mathrm{x}, \mathrm{y}, \mathrm{z}) \in \mathrm{R}^{2} \times[-\mathrm{h}, 0]$, subjected to the following boundary conditions

$$
\begin{array}{lll}
\phi_{\mathrm{z}}=\eta_{\mathrm{t}} & \text { on } & \mathrm{z}=0 \\
\phi_{\mathrm{z}}=\zeta_{\mathrm{t}} & \text { on } & \mathrm{z}=-\mathrm{h} \\
\phi_{\mathrm{t}}+\mathrm{g} \eta=0 & \text { on } & \mathrm{z}=0
\end{array}
$$

The linearized shallow water solution can be obtained by the Fourier-Laplace transform.

\subsection{Solution of the Problem}

Our interest is the resulting uplift of the free surface elevation $\eta(\mathrm{x}, \mathrm{y}, \mathrm{t})$. An analytical analyses is examine to illustrate the generation and propagation of a tsunami for a given bed profile $\zeta(\mathrm{x}, \mathrm{y}, \mathrm{t})$. Mathematical modeling of waves generated by vertical and lateral displacements of ocean bottom using the combined Fourier-Laplace transform of the Laplace equation analytically is the simplest way of studying tsunami development. All our studies were taken into account constant depths for which the Laplace and Fast Fourier Transform (FFT) methods could be applied. The Equations (6)-(9) can be solved by using the method of integral transforms. We apply the Fourier transform in $(\mathrm{x}, \mathrm{y})$.

$$
\mathfrak{F}[f]=\hat{f}\left(\mathrm{k}_{1}, \mathrm{k}_{2}\right)=\int_{\mathrm{R}^{2}} \mathrm{f}(\mathrm{x}, \mathrm{y}) \mathrm{e}^{-\mathrm{i}\left(\mathrm{k}_{1} \mathrm{x}+\mathrm{k}_{2} \mathrm{y}\right)} \mathrm{dx} \mathrm{dy}
$$

with its inverse transform

$$
\mathfrak{F}^{-1}[\widehat{f}]=f(\mathrm{x}, \mathrm{y})=\frac{1}{(2 \pi)^{2}} \int_{\mathrm{R}^{2}} \hat{f}\left(\mathrm{k}_{1}, \mathrm{k}_{2}\right) \mathrm{e}^{\mathrm{i}\left(\mathrm{k}_{1} \mathrm{x}+\mathrm{k}_{2} \mathrm{y}\right)} \mathrm{dk}_{1} \mathrm{dk}_{2}
$$

and the Laplace transform in time t,

$$
£[g]=\mathrm{G}(\mathrm{s})=\int_{0}^{\infty} \mathrm{g}(\mathrm{t}) \mathrm{e}^{-\mathrm{st}} \mathrm{dt}
$$

For the combined Fourier and Laplace transforms, the following notation is introduced:

$\mathfrak{F}(£(f(x, y, t))=$

$$
\overline{\mathrm{F}}\left(\mathrm{k}_{1}, \mathrm{k}_{2}, \mathrm{~s}\right)=\int_{\mathrm{R}^{2}} \mathrm{e}^{-\mathrm{i}\left(\mathrm{k}_{1} \mathrm{x}+\mathrm{k}_{2} \mathrm{y}\right)} \mathrm{dx} d \mathrm{dy} \int_{0}^{\infty} \mathrm{f}(\mathrm{x}, \mathrm{y}, \mathrm{t}) \mathrm{e}^{-\mathrm{st}} \mathrm{dt}
$$

Combining (7) and (9) yields the single free-surface condition

$$
\phi_{\mathrm{tt}}(\mathrm{x}, \mathrm{y}, 0, \mathrm{t})+g \phi_{\mathrm{z}}(\mathrm{x}, \mathrm{y}, 0, \mathrm{t})=0
$$

After applying the transforms and using the property $\mathfrak{F}\left[\frac{d^{n} f}{d x^{n}}\right]=(\mathrm{ik})^{\mathrm{n}} \overline{\mathrm{F}}(\mathrm{k})$ and the initial conditions (5), Equations (6), (8) and (10) become

$$
\begin{gathered}
\bar{\phi}_{\mathrm{zz}}\left(\mathrm{k}_{1}, \mathrm{k}_{2}, \mathrm{z}, \mathrm{s}\right)-\left(\mathrm{k}_{1}^{2}+\mathrm{k}_{2}{ }^{2}\right) \bar{\phi}\left(\mathrm{k}_{1}\right), \mathrm{k}_{2}, \mathrm{z}, \mathrm{s}=0 \\
\bar{\phi}_{\mathrm{z}}\left(\mathrm{k}_{1}, \mathrm{k}_{2},-\mathrm{h}, \mathrm{s}\right)=\mathrm{s} \bar{\zeta}\left(\mathrm{k}_{1}, \mathrm{k}_{2}, \mathrm{~s}\right) \\
\mathrm{s}^{2} \bar{\phi}\left(\mathrm{k}_{1}, \mathrm{k}_{2}, 0, \mathrm{~s}\right)+\mathrm{g} \bar{\phi}_{\mathrm{z}}\left(\mathrm{k}_{1}, \mathrm{k}_{2}, 0, \mathrm{~s}\right)=0
\end{gathered}
$$

The transformed free-surface elevation can be ob- tained from (9) as

$$
\bar{\eta}\left(\mathrm{k}_{1}, \mathrm{k}_{2}, \mathrm{~s}\right)=-\frac{\mathrm{s}}{\mathrm{g}} \bar{\phi}\left(\mathrm{k}_{1}, \mathrm{k}_{2}, 0, \mathrm{~s}\right)
$$

A general solution of (11) will be given by

$$
\bar{\phi}\left(\mathrm{k}_{1}, \mathrm{k}_{2}, \mathrm{z}, \mathrm{s}\right)=\mathrm{A}\left(\mathrm{k}_{1}, \mathrm{k}_{2}, \mathrm{~s}\right) \cosh (\mathrm{kz})+\mathrm{B}\left(\mathrm{k}_{1}, \mathrm{k}_{2}, \mathrm{~s}\right) \sinh (\mathrm{kz})
$$

where $\mathrm{k}=\sqrt{\mathrm{k}_{1}{ }^{2}+\mathrm{k}_{2}{ }^{2}}$. The functions $\mathrm{A}\left(\mathrm{k}_{1}, \mathrm{k}_{2}, \mathrm{~s}\right)$ and $\mathrm{B}\left(\mathrm{k}_{1}, \mathrm{k}_{2}, \mathrm{~s}\right)$ can be easily found from the boundary conditions (12) and (13),

$$
\begin{aligned}
& \mathrm{A}\left(\mathrm{k}_{1}, \mathrm{k}_{2}, \mathrm{~s}\right)=-\frac{\mathrm{gs} \bar{\zeta}\left(\mathrm{k}_{1}, \mathrm{k}_{2}, \mathrm{~s}\right)}{\cosh (\mathrm{kh})\left[\mathrm{s}^{2}+\mathrm{gk} \tanh (\mathrm{kh})\right]} \\
& \mathrm{B}\left(\mathrm{k}_{1}, \mathrm{k}_{2}, \mathrm{~s}\right)=\frac{\mathrm{s}^{3} \bar{\zeta}\left(\mathrm{k}_{1}, \mathrm{k}_{2}, \mathrm{~s}\right)}{\mathrm{k} \cosh (\mathrm{kh})\left[\mathrm{s}^{2}+\mathrm{gk} \tanh (\mathrm{kh})\right]}
\end{aligned}
$$

Substituting the expressions for the functions A and B in the general solution (15) yields

$$
\bar{\phi}\left(\mathrm{k}_{1}, \mathrm{k}_{2}, \mathrm{z}, \mathrm{s}\right)=-\frac{\mathrm{gs} \bar{\zeta}\left(\mathrm{k}_{1}, \mathrm{k}_{2}, \mathrm{~s}\right)}{\cosh (\mathrm{kh})\left[\mathrm{s}^{2}+\omega^{2}\right]}\left(\cosh (\mathrm{kz})-\frac{\mathrm{s}^{2}}{\mathrm{gk}} \sinh (\mathrm{kz})\right)
$$

where $\omega=\sqrt{\mathrm{gk} \tanh (\mathrm{kh})}$ is the circular frequency of the wave motion. The free surface elevation $\bar{\eta}\left(\mathrm{k}_{1}, \mathrm{k}_{2}, \mathrm{~s}\right)$ can be obtained from (14) as

$$
\bar{\eta}\left(\mathrm{k}_{1}, \mathrm{k}_{2}, \mathrm{~s}\right)=\frac{\mathrm{s}^{2} \bar{\zeta}\left(\mathrm{k}_{1}, \mathrm{k}_{2}, \mathrm{~s}\right)}{\cosh (\mathrm{kh})\left(\mathrm{s}^{2}+\omega^{2}\right)}
$$

A solution for $\eta(\mathrm{x}, \mathrm{y}, \mathrm{t})$ can be evaluated for specified $\zeta(\mathrm{x}, \mathrm{y}, \mathrm{t})$ by computing approximately its transform $\bar{\zeta}\left(\mathrm{k}_{1}, \mathrm{k}_{2}, \mathrm{~s}\right)$ then substituting it into (17) and inverting $\bar{\eta}\left(\mathrm{k}_{1}, \mathrm{k}_{2}, \mathrm{~s}\right)$ to obtain $\bar{\eta}\left(\mathrm{k}_{1}, \mathrm{k}_{2}, \mathrm{t}\right)$. We concern to evaluate $\eta(\mathrm{x}, \mathrm{y}, \mathrm{t})$ by transforming analytically the assumed source model then inverting the Laplace transform of $\bar{\eta}\left(\mathrm{k}_{1}, \mathrm{k}_{2}, \mathrm{~s}\right)$ to obtain $\bar{\eta}\left(\mathrm{k}_{1}, \mathrm{k}_{2}, \mathrm{t}\right)$ which is further converted to $\eta(\mathrm{x}, \mathrm{y}, \mathrm{t})$ by using double inverse Fourier Transform.

The circular frequency $\omega$ describes the dispersion relation of tsunamis and implies phase velocity $\mathrm{c}=\frac{\omega}{\mathrm{k}}$ and group velocity $\mathrm{U}=\frac{\mathrm{d} \omega}{\mathrm{dk}}$. Hence, $\mathrm{c}=\sqrt{\frac{\mathrm{g} \tanh (\mathrm{kh})}{\mathrm{k}}}$, and $\mathrm{U}=\frac{1}{2} \mathrm{c}\left(1+\frac{2 \mathrm{kh}}{\sinh (2 \mathrm{kz})}\right) \cdot$

Since, $\mathrm{k}=\frac{2 \pi}{\lambda}$, hence as $\mathrm{kh} \rightarrow 0$, both $\mathrm{c} \rightarrow \sqrt{\mathrm{gh}}$ and $\mathrm{U} \rightarrow \sqrt{\mathrm{gh}}$, which implies that the tsunami velocity $\mathrm{V}_{\mathrm{t}}=$ $\sqrt{\mathrm{gh}}$ for wavelengths $\lambda$ long compared to the water depth $h$. The above linearized solution is known as the shallow water solution. We considered two models for 
the sea floor displacement, namely, a slowly curvilinear vertical faulting with rise time $0 \leq \mathrm{t} \leq \mathrm{t}_{1}$ and a variable single slip-fault, propagating unilaterally in the positive $\mathrm{x}$-direction with time $\mathrm{t}_{1} \leq \mathrm{t} \leq \mathrm{t}^{*}$, both with finite velocity $\mathrm{v}$. In the-direction, the models propagate instant a- neously. The set of physical parameters used in the problem are given in Table 1.

The two models are shown in Figures 2 and $\mathbf{3}$, respectively, and given by:

a) Slowly curvilinear uplift faulting

Table 1. Parameters used in the analytical solution of the problem.

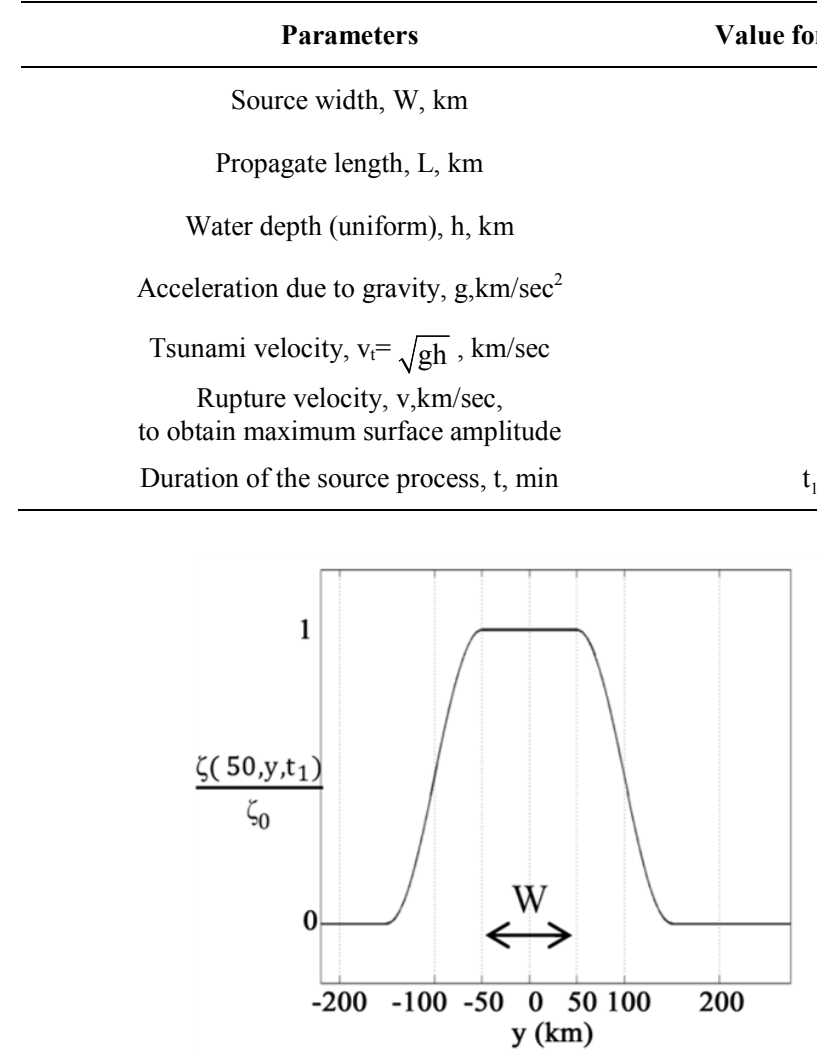

(a)

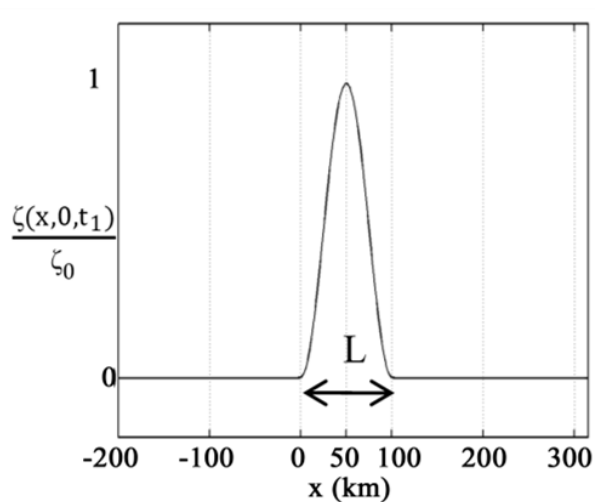

(b)

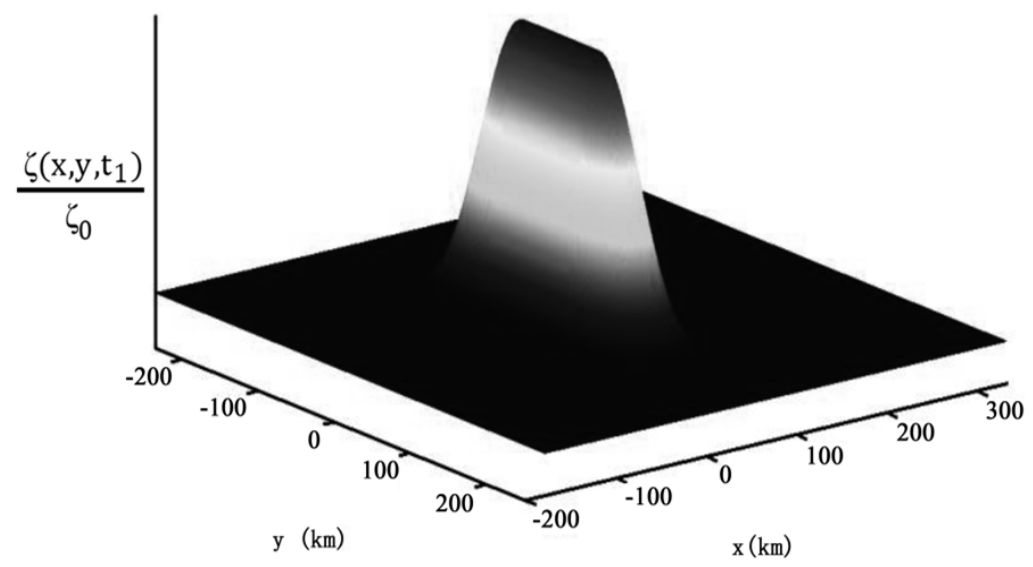

(c)

Figure 2. Normalized bed deformation representing by a slowly curvilinear uplift faulting at $t_{1}=\frac{50}{v}$ (a) Side view along the axis of the symmetry at $\mathrm{x}=50$; (b) Side view along the axis of the symmetry at $y=0$; (c) Three- dimensional view. 


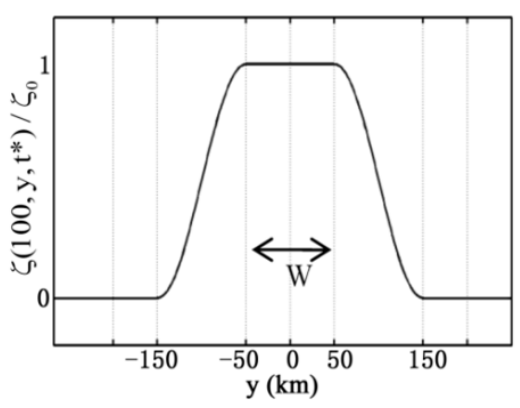

(a)

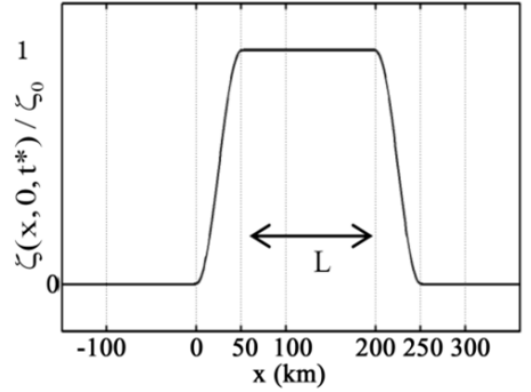

(b)

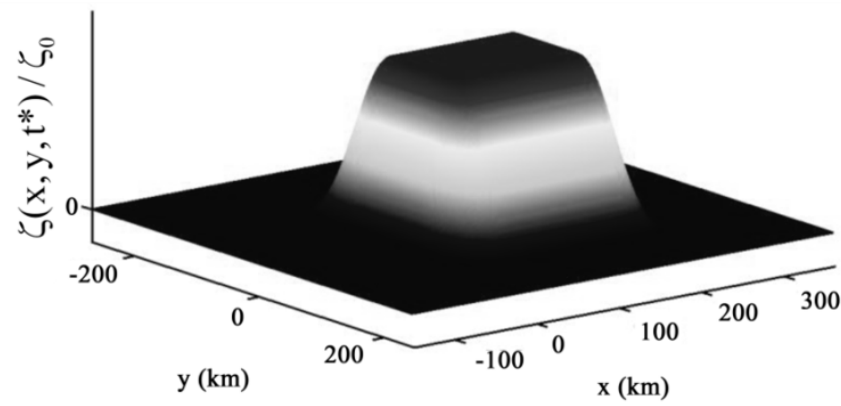

(c)

Figure 3. Normalized Bed deformation model representing by a single-fault slip at $t=t *=200 / v$. (a) Side view along the axis of the symmetry at $x=100$; (b) Side view along the axis of the symmetry at $y=0$;(c) Three-dimensional view.

$$
\zeta(x, y, t)=\left\{\begin{array}{cc}
\zeta_{0} \frac{\mathrm{vt}}{2 \mathrm{~L}}\left(1-\cos \frac{\pi}{50} \mathrm{x}\right)\left[1-\cos \frac{\pi}{100}(\mathrm{y}+150)\right], & 0 \leq \mathrm{x} \leq 100, \quad-150 \leq \mathrm{y} \leq-50 \\
\zeta_{0} \frac{\mathrm{vt}}{\mathrm{L}}\left(1-\cos \frac{\pi}{50} \mathrm{x}\right), & 0 \leq \mathrm{x} \leq 100, \quad-50 \leq \mathrm{y} \leq 50 \\
\zeta_{0} \frac{\mathrm{vt}}{2 \mathrm{~L}}\left(1-\cos \frac{\pi}{50} \mathrm{x}\right)\left[1+\cos \frac{\pi}{100}(\mathrm{y}-150)\right], & 0 \leq \mathrm{x} \leq 100, \quad 50 \leq \mathrm{y} \leq 150
\end{array}\right.
$$

For this displacement, the bed rises during $0 \leq \mathrm{t} \leq \mathrm{t}_{1}$ to a maximum displacement $\zeta_{0}$ in an asymptotic manner.

b) Curvilinear slip-fault

$$
\zeta(\mathrm{x}, \mathrm{y}, \mathrm{t})=\zeta_{1}(\mathrm{x}, \mathrm{y}, \mathrm{t})+\zeta_{2}(\mathrm{x}, \mathrm{y}, \mathrm{t})+\zeta_{3}(\mathrm{x}, \mathrm{y}, \mathrm{t}),
$$

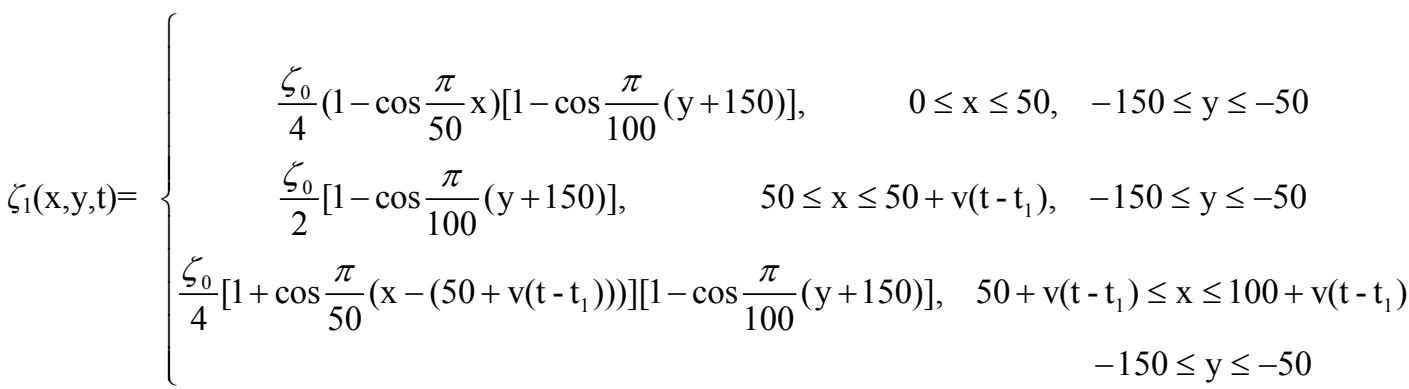

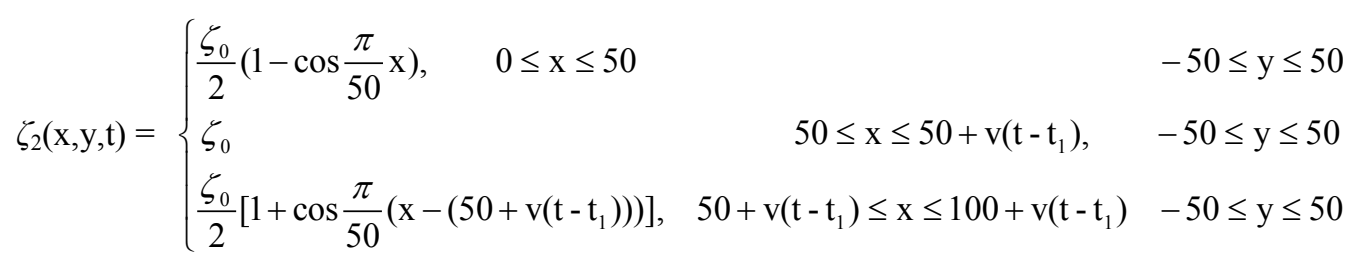




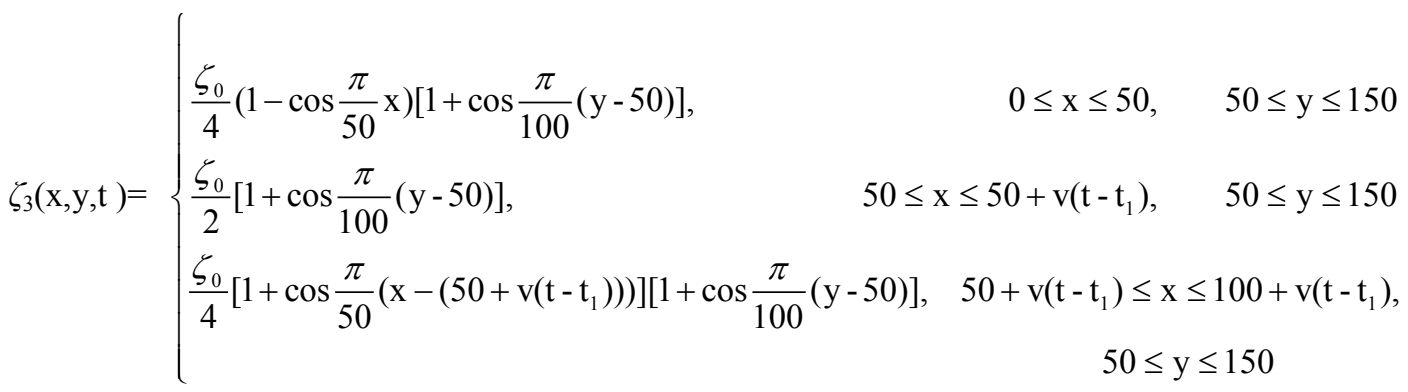

For this source model, the free surface elevation takes initially the deformation of the bed shown in Figure 2 which remain at this elevation $\boldsymbol{\zeta}_{\mathbf{0}}$ for $\mathbf{t} \geq \mathbf{t}_{\mathbf{1}}$ and further propagate unilaterally in the positive $\mathrm{x}$ - direction with velocity $\mathrm{v}$ till it reaches $\mathbf{t}^{*}$.
Laplace and Fourier transform can now be applied to the bed motion described by (18) and (19). First, beginning with the uplift faulting (18) for $\underline{\theta} \mathrm{t} \leq \mathrm{t}_{1}$ where $\mathrm{t}_{1}=\frac{50}{\mathrm{v}}$ and

$$
\mathfrak{F}\left(£(\zeta(\mathrm{x}, \mathrm{y}, \mathrm{t}))=\bar{\zeta}\left(\mathrm{k}_{1}, \mathrm{k}_{2}, \mathrm{~s}\right)=\int_{-\infty}^{\infty} \mathrm{e}^{-\mathrm{i}\left(\mathrm{k}_{1} \mathrm{x}+\mathrm{k}_{2} \mathrm{y}\right)} \mathrm{dx} \mathrm{dy} \int_{0}^{\infty} \zeta(\mathrm{x}, \mathrm{y}, \mathrm{t}) \mathrm{e}^{-\mathrm{st}} \mathrm{dt}\right.
$$

The limits of the above integration are apparent from (18).

$$
\begin{aligned}
& \bar{\eta}\left(\mathrm{k}_{1}, \mathrm{k}_{2}, \mathrm{~s}\right)= \\
& \frac{\mathrm{s}}{\cosh (\mathrm{kh})\left(\mathrm{s}^{2}+\omega^{2}\right)} \zeta_{0} \frac{\mathrm{v}}{\mathrm{L}} \frac{1}{2 \mathrm{~s}}\left[\frac{\left(1-\mathrm{e}^{-\mathrm{i} 100 \mathrm{k}_{1}}\right)}{\mathrm{ik}_{1}}-\frac{\mathrm{e}^{-\mathrm{i} 100 \mathrm{k}_{1}}}{1-\left(\frac{50}{\pi} \mathrm{k}_{1}\right)^{2}}\left[\mathrm{ik}_{1}\left(\frac{50}{\pi}\right)^{2}\left(\mathrm{e}^{\mathrm{i} 100 \mathrm{k}_{1}}-1\right)\right]\right] \times \\
& {\left[\begin{array}{c}
\left.\frac{\left(\mathrm{e}^{\mathrm{i} 150 \mathrm{k}_{2}}-\mathrm{e}^{\mathrm{i} 50 \mathrm{k}_{2}}\right)}{\mathrm{ik} \mathrm{k}_{2}}-\frac{1}{1-\left(\frac{100}{\pi} \mathrm{k}_{2}\right)^{2}}\left[\mathrm{ik}_{2}\left(\frac{100}{\pi}\right)^{2}\left(\mathrm{e}^{\mathrm{i} 50 \mathrm{k}_{2}}+\mathrm{e}^{\mathrm{i} 150 \mathrm{k}_{2}}\right)\right]+\frac{4 \sin \left(50 \mathrm{k}_{2}\right)}{\mathrm{k}_{2}}+\right] \\
{\left[\frac{\left(\mathrm{e}^{-\mathrm{i} 50 \mathrm{k}_{2}}-\mathrm{e}^{-\mathrm{i} 150 \mathrm{k}_{2}}\right)}{\mathrm{ik}_{2}}+\frac{1}{1-\left(\frac{100}{\pi} \mathrm{k}_{2}\right)^{2}}\right]\left[\mathrm{ik}_{2}\left(\frac{100}{\pi}\right)^{2}\left(\mathrm{e}^{\mathrm{i} 50 \mathrm{k}_{2}}+\mathrm{e}^{\mathrm{i} 150 \mathrm{k}_{2}}\right)\right]}
\end{array}\right]}
\end{aligned}
$$

The free surface elevation $\bar{\eta}\left(\mathrm{k}_{1}, \mathrm{k}_{2}, \mathrm{t}\right)$ can be evaluated by using inverse Laplace transform of $\bar{\eta}\left(\mathrm{k}_{1}, \mathrm{k}_{2}, \mathrm{~s}\right)$ as follows:

First, recall that $£^{-1}\left(\frac{\mathrm{s}}{\mathrm{s}^{2}+\omega^{2}}\right)=\cos \omega \mathrm{t}$ and $£^{-1}\left(\frac{1}{\mathrm{~s}}\right)=1$,

$$
\begin{aligned}
& \bar{\eta}\left(\mathrm{k}_{1}, \mathrm{k}_{2}, \mathrm{t}\right)=\frac{\sin \omega \mathrm{t}}{\omega \cosh (\mathrm{kh})} \frac{\zeta_{0} \mathrm{v}}{2 \mathrm{~L}}\left[\frac{\left(1-\mathrm{e}^{-\mathrm{i} 100 \mathrm{k}_{1}}\right)}{\mathrm{ik}_{1}}-\frac{\mathrm{e}^{-\mathrm{i} 100 \mathrm{k}_{1}}}{1-\left(\frac{50}{\pi} \mathrm{k}_{1}\right)^{2}}\left[\mathrm{ik}_{1}\left(\frac{50}{\pi}\right)^{2}\left(\mathrm{e}^{-\mathrm{i} 100 \mathrm{k}_{1}}-1\right]\right] \times\right. \\
& {\left[\begin{array}{c}
\frac{\mathrm{e}^{\mathrm{i} 150 \mathrm{k}_{2}}-\mathrm{e}^{\mathrm{i} 50 \mathrm{k}_{2}}}{\mathrm{ik} \mathrm{k}_{2}}-\frac{1}{1-\left(\frac{100}{\pi} \mathrm{k}_{2}\right)^{2}}\left[\mathrm{ik}_{2}\left(\frac{100}{\pi}\right)^{2}\left(\mathrm{e}^{\mathrm{i} 50 \mathrm{k}_{2}}+\mathrm{e}^{\mathrm{i} 150 \mathrm{k}_{2}}\right)\right]+\frac{4 \sin \left(50 \mathrm{k}_{2}\right)}{\mathrm{k}_{2}}+ \\
{\left[\frac{\left(\mathrm{e}^{-\mathrm{i} 50 \mathrm{k}_{2}}-\mathrm{e}^{-\mathrm{i} 150 \mathrm{k}_{2}}\right)}{\mathrm{ik}_{2}}+\frac{1}{1-\left(\frac{100}{\pi} \mathrm{k}_{2}\right)^{2}}\right]\left[\mathrm{ik}_{2}\left(\frac{100}{\pi}\right)^{2}\left(\mathrm{e}^{-\mathrm{i} 150 \mathrm{k}_{2}}+\mathrm{e}^{-\mathrm{i} 50 \mathrm{k}_{2}}\right)\right]}
\end{array}\right]}
\end{aligned}
$$


In case for $\mathrm{t} \geq \mathrm{t}_{1}, \bar{\eta}\left(\mathrm{k}_{1}, \mathrm{k}_{2}, \mathrm{t}\right)$ will have the same expression except in the convolution step, the integral become $\int_{t-t_{1}}^{t} \cos \omega \tau \mathrm{d} \tau=\frac{\sin \omega \mathrm{t}}{\omega}-\frac{\sin \omega\left(\mathrm{t}-\mathrm{t}_{1}\right)}{\omega}$

Finally, $\eta(\mathrm{x}, \mathrm{y}, \mathrm{t})$ is evaluated using the double inverse Fourier transform of $\bar{\eta}\left(\mathrm{k}_{1}, \mathrm{k}_{2}, \mathrm{t}\right)$

$$
\eta(\mathrm{x}, \mathrm{y}, \mathrm{t})=\frac{1}{(2 \pi)^{2}} \int_{-\infty}^{\infty} e^{\mathrm{i} \mathrm{k}_{2} \mathrm{y}}\left[\int_{-\infty}^{\infty} e^{\mathrm{i} \mathrm{k}_{1} \mathrm{x}} \bar{\eta}\left(\mathrm{k}_{1}, \mathrm{k}_{2}, \mathrm{t}\right) \mathrm{dk}_{1}\right] \mathrm{dk}_{2}
$$

This inversion is computed by using the FFT. The inverse FFT is a fast algorithm for efficient implementation of the Inverse Discrete Fourier Transform (IDFT) given by

$$
f(m, n)=\frac{1}{M N} \sum_{p=0}^{M-1} \sum_{q=0}^{N-1} F(p, q) e^{i\left(\frac{2 \pi}{M}\right) p m} e^{i\left(\frac{2 \pi}{N}\right) q n}, \quad \begin{gathered}
p=0,1, \ldots \ldots, M-1 \\
q=0,1, \ldots \ldots . N-1
\end{gathered}
$$

where $f(m, n)$ is the resulted function of the two spatial variables $\mathrm{m}$ and $\mathrm{n}$,corresponding $\mathrm{x}$ and $\mathrm{y}$, from the frequency domain function $\mathrm{F}(\mathrm{p}, \mathrm{q})$ with frequency variables $\mathrm{p}$ and $\mathrm{q}$, corresponding $\mathrm{k}_{1}$ and $\mathrm{k}_{2}$. This inversion is done efficiently by using the Matlab FFT algorithm.

In order to implement the algorithm efficiently, singularities should be removed by finite limits as follows:

1) As $\mathrm{k} \rightarrow 0$, implies $\mathrm{k}_{1} \rightarrow 0, \mathrm{k}_{2} \rightarrow 0$ and $\omega \rightarrow 0$ then $\bar{\eta}\left(\mathrm{k}_{1}, \mathrm{k}_{2}, \mathrm{t}\right)$ has the following limit

$\lim _{\mathrm{k} \rightarrow 0} \bar{\eta}\left(\mathrm{k}_{1}, \mathrm{k}_{2}, \mathrm{t}\right)=\left\{\begin{array}{ll}200 \zeta_{0} \mathrm{vt} & \mathrm{t} \leq \mathrm{t}_{1} \\ 200 \zeta_{0} \mathrm{vt}_{1} & \mathrm{t} \geq \mathrm{t}_{1}\end{array}\right.$, where $\mathrm{t}_{1}=\frac{50}{\mathrm{v}}$
2) As $\mathrm{k}_{2} \rightarrow 0$, then the singular terms of $\bar{\eta}\left(\mathrm{k}_{1}, \mathrm{k}_{2}, \mathrm{t}\right)$ have the following limits

$$
\begin{gathered}
\lim _{\mathrm{k}_{2} \rightarrow 0} \mathrm{k}_{2}\left(\frac{\mathrm{e}^{\mathrm{i} 150 \mathrm{k}_{2}}}{\mathrm{i} \mathrm{k_{2 }}}-\frac{\mathrm{e}^{\mathrm{i} 50 \mathrm{k}_{2}}}{\mathrm{i} \mathrm{k_{2 }}}\right)=100 \\
\lim _{\mathrm{k}_{2} \rightarrow 0}\left(\frac{4 \sin \left(50 \mathrm{k}_{2}\right)}{\mathrm{k}_{2}}\right)=200 \\
\lim _{\mathrm{k}_{2} \rightarrow 0}\left(\frac{\mathrm{e}^{-\mathrm{i} 50 \mathrm{k}_{2}}}{\mathrm{i} \mathrm{k}_{2}}-\frac{\mathrm{e}^{-\mathrm{i} 150 \mathrm{k}_{2}}}{\mathrm{i} \mathrm{k_{2 }}}\right)=100
\end{gathered}
$$

3) As $\mathrm{k}_{1} \rightarrow 0$, then the singular term of $\bar{\eta}\left(\mathrm{k}_{1}, \mathrm{k}_{2}, \mathrm{t}\right)$ has the following limit

$$
\lim _{\mathrm{k}_{1} \rightarrow 0}\left(\frac{1}{\mathrm{ik}_{2}}-\frac{\mathrm{e}^{-\mathrm{i} 100 \mathrm{k}_{1}}}{\mathrm{i} \mathrm{k_{1 }}}\right)=100
$$

Using the same steps, $\bar{\eta}\left(\mathrm{k}_{1}, \mathrm{k}_{2}, \mathrm{t}\right)$ is evaluated by applying the Laplace and Fourier transform to the bed motion described by (19), then substituting into (17) and then inverting the Laplace transform on $\bar{\eta}\left(\mathrm{k}_{1}, \mathrm{k}_{2}, \mathrm{~s}\right)$ to obtain $\bar{\eta}\left(\mathrm{k}_{1}, \mathrm{k}_{2}, \mathrm{t}\right)$. This is verified for $\mathrm{t}_{1} \leq \mathrm{t} \leq \mathrm{t}^{*}$ where $\mathrm{t}^{*}=\frac{200}{\mathrm{v}}$ as follows:

$$
\bar{\eta}\left(\mathrm{k}_{1}, \mathrm{k}_{2}, t\right)=\overline{\eta_{1}}\left(\mathrm{k}_{1}, \mathrm{k}_{2}, t\right)+\overline{\eta_{2}}\left(\mathrm{k}_{1}, \mathrm{k}_{2}, t\right)+\overline{\eta_{3}}\left(\mathrm{k}_{1}, \mathrm{k}_{2}, t\right)
$$

where, $\overline{\eta_{1}}\left(\mathrm{k}_{1}, \mathrm{k}_{2}, \mathrm{t}\right), \overline{\eta_{2}}\left(\mathrm{k}_{1}, \mathrm{k}_{2}, \mathrm{t}\right)$ and $\overline{\eta_{3}}\left(\mathrm{k}_{1}, \mathrm{k}_{2}, \mathrm{t}\right)$ can be written respectively as:

$$
\begin{aligned}
& \overline{\eta_{1}}\left(\mathrm{k}_{1}, \mathrm{k}_{2}, \mathrm{t}\right)= \\
& {\left[\frac{\zeta_{0}}{4 \cosh (k h)}\right]\left[\frac{\mathrm{e}^{\mathrm{i} 150 \mathrm{k}_{2}}-\mathrm{e}^{\mathrm{i} 50 \mathrm{k}_{2}}}{\mathrm{i} \mathrm{k_{2 }}}-\frac{1}{1-\left(\frac{100}{\pi} \mathrm{k}_{2}\right)^{2}}\left[\mathrm{ik}_{2}\left(\frac{100}{\pi}\right)^{2}\left(\mathrm{e}^{\mathrm{i} 50 \mathrm{k}_{2}}+\mathrm{e}^{\mathrm{i} 150 \mathrm{k}_{2}}\right)\right] \times\right.}
\end{aligned}
$$

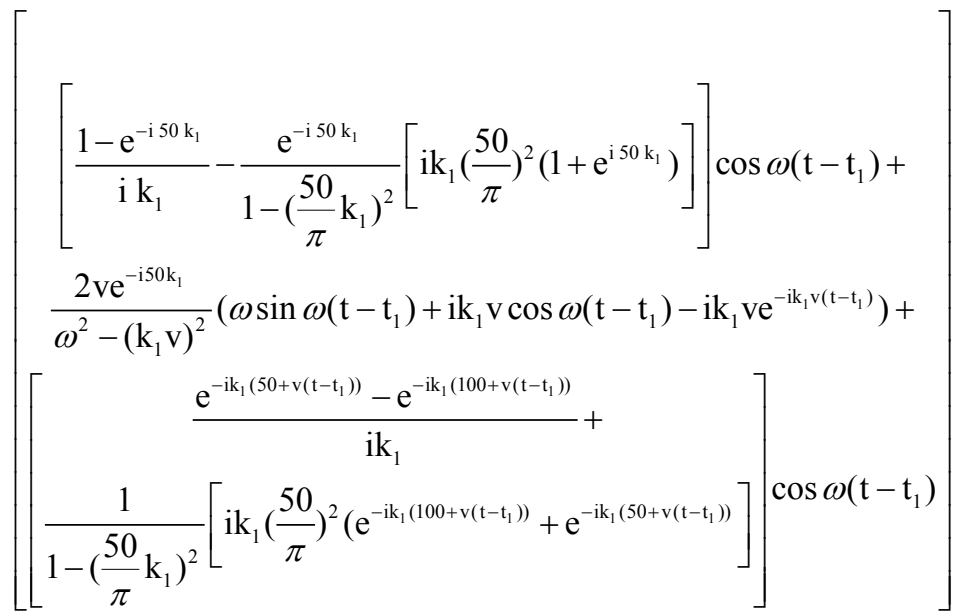




$$
\begin{aligned}
& \overline{\eta_{2}}\left(\mathrm{k}_{1}, \mathrm{k}_{2}, \mathrm{t}\right)=\left[\frac{\zeta_{0}}{\cosh (\mathrm{kh})}\right]\left[\frac{\sin \left(50 \mathrm{k}_{2}\right)}{\mathrm{k}_{2}}\right] \times
\end{aligned}
$$

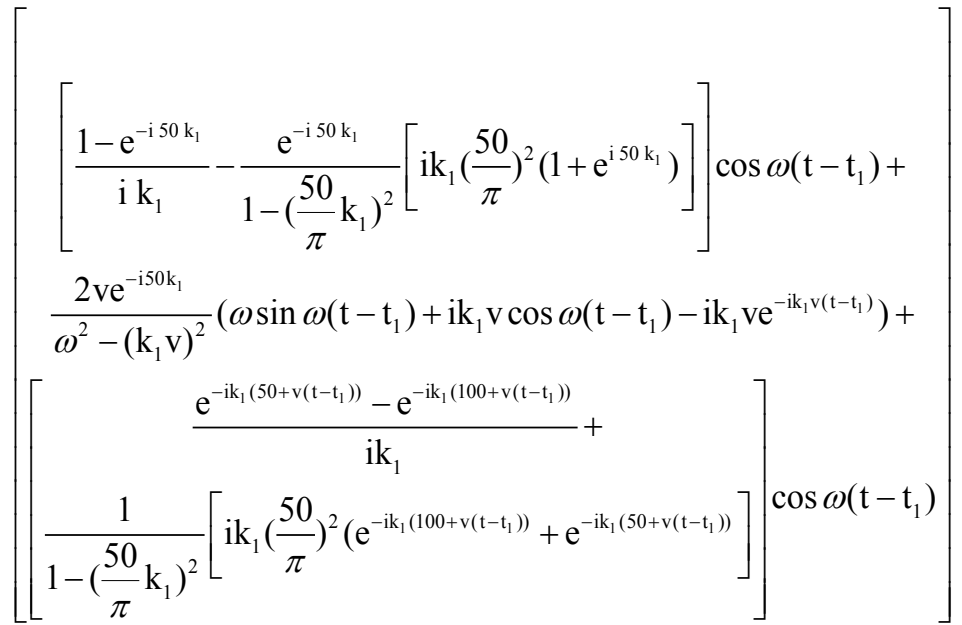

and

$$
\overline{\eta_{3}}\left(\mathrm{k}_{1}, \mathrm{k}_{2}, \mathrm{t}\right)=\left[\frac{\zeta_{0}}{4 \cosh (\mathrm{kh})}\right]\left[\frac{\mathrm{e}^{-\mathrm{i} 50 \mathrm{k}_{2}}-\mathrm{e}^{-\mathrm{i} 150 \mathrm{k}_{2}}}{\mathrm{i} \mathrm{k}_{2}}+\frac{1}{1-\left(\frac{100}{\pi} \mathrm{k}_{2}\right)^{2}}\left[\mathrm{ik}_{2}\left(\frac{100}{\pi}\right)^{2}\left(\mathrm{e}^{-\mathrm{i} 150 \mathrm{k}_{2}}+\mathrm{e}^{-\mathrm{i} 50 \mathrm{k}_{2}}\right]\right] \times\right.
$$

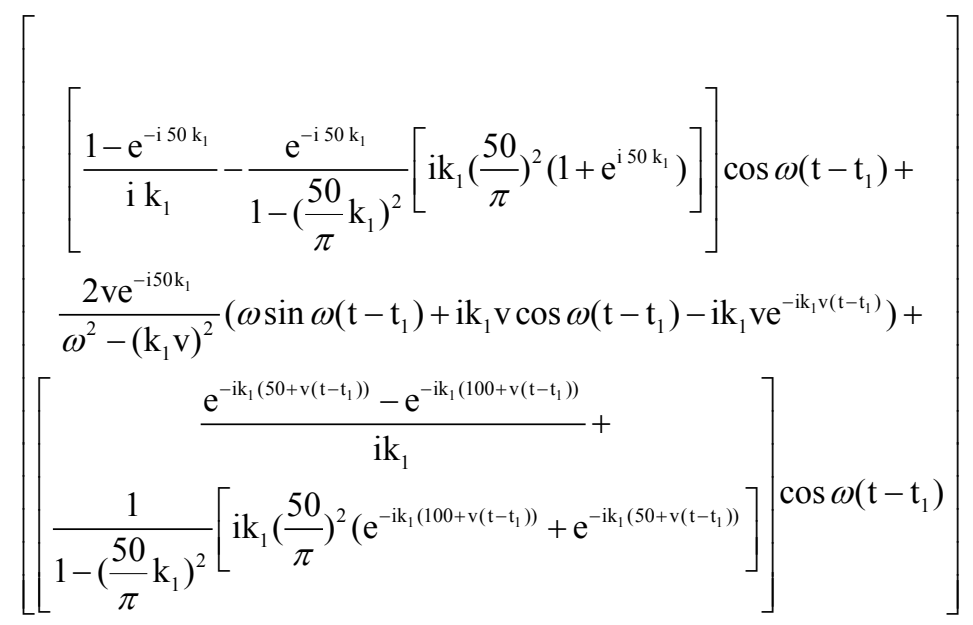

Substituting $\overline{\eta_{1}}\left(\mathrm{k}_{1}, \mathrm{k}_{2}, \mathrm{t}\right), \overline{\eta_{2}}\left(\mathrm{k}_{1}, \mathrm{k}_{2}, \mathrm{t}\right)$ and $\overline{\eta_{3}}\left(\mathrm{k}_{1}, \mathrm{k}_{2}, \mathrm{t}\right)$ into $(24)$ gives $\eta\left(\mathrm{k}_{1}, \mathrm{k}_{2}, \mathrm{t}\right)$ for $\mathrm{t}_{1} \leq \mathrm{t} \leq \mathrm{t}^{*}$
In case for $\mathrm{t} \geq \mathrm{t}^{*}, \bar{\eta}\left(\mathrm{k}_{1}, \mathrm{k}_{2}, \mathrm{t}\right)$ will have the expression (24) except the term resulting from the convolution theorem, i.e.

$$
\int_{\left(\mathrm{t}-\mathrm{t}_{1}\right)-\mathrm{t}^{*}}^{\mathrm{t}} \cos \omega \tau \mathrm{e}^{-\mathrm{i} \mathrm{k}_{1} \mathrm{v}\left(\mathrm{t}-\mathrm{t}_{1}\right)-\tau} \mathrm{d} \tau=\frac{1}{\omega^{2}-\left(\mathrm{k}_{1} \mathrm{v}\right)^{2}}\left[\begin{array}{l}
\omega \sin \omega\left(\mathrm{t}-\mathrm{t}_{1}\right)+\mathrm{ik}_{1} \mathrm{v} \cos \omega\left(\mathrm{t}-\mathrm{t}_{1}\right) \\
-\mathrm{e}^{-\mathrm{ik}_{1} \mathrm{vt}^{*}}\left(\omega \sin \omega\left(\left(\mathrm{t}-\mathrm{t}_{1}\right)-\mathrm{t}^{*}\right)+\mathrm{ik}_{1} \mathrm{v} \cos \omega\left(\left(\mathrm{t}-\mathrm{t}_{1}\right)-\mathrm{t}^{*}\right)\right)
\end{array}\right],
$$

instead of

$$
\int_{\left(\mathrm{t}-\mathrm{t}_{1}\right)-\mathrm{t}^{*}}^{\mathrm{t}} \cos \omega \tau \mathrm{e}^{-\mathrm{ik} \mathrm{k}_{1} \mathrm{v}(\mathrm{t}-\tau)} \mathrm{d} \tau=\frac{1}{\omega^{2}-\left(\mathrm{k}_{1} \mathrm{v}\right)^{2}}\left(\omega \sin \omega\left(\mathrm{t}-\mathrm{t}_{1}\right)+\mathrm{ik}_{1} \mathrm{v} \cos \omega\left(\mathrm{t}-\mathrm{t}_{1}\right)-\mathrm{ik}_{1} \mathrm{ve}^{-\mathrm{i} \mathrm{k}_{1} \mathrm{v}\left(\mathrm{t}-\mathrm{t}_{1}\right)}\right)
$$


Finally, $\eta(\mathrm{x}, \mathrm{y}, \mathrm{t})$ is computed using inverse Fast Fourier transform of $\bar{\eta}\left(\mathrm{k}_{1}, \mathrm{k}_{2}, \mathrm{t}\right)$.Again, the singular points should be removed to compute $\eta(\mathrm{x}, \mathrm{y}, \mathrm{t})$ efficiently

1) As $\mathrm{k} \rightarrow 0$, then $\bar{\eta}\left(\mathrm{k}_{1}, \mathrm{k}_{2}, \mathrm{t}\right)$ has the following limit

$\lim _{\mathrm{k} \rightarrow 0} \bar{\eta}\left(\mathrm{k}_{1}, \mathrm{k}_{2}, \mathrm{t}\right)= \begin{cases}2 \zeta_{0}\left(5000+100 \mathrm{v}\left(\mathrm{t}-\mathrm{t}_{1}\right)\right. & \left(\mathrm{t}-\mathrm{t}_{1}\right) \leq \mathrm{t} * \\ 2 \zeta_{0}(5000+100 \mathrm{vt} * & \left(\mathrm{t}-\mathrm{t}_{1}\right) \geq \mathrm{t}^{*}\end{cases}$ where $\mathrm{t}^{*}=\frac{200}{\mathrm{v}}$.

2) As $\mathrm{k}_{2} \rightarrow 0$, then the singular terms of $\bar{\eta}\left(\mathrm{k}_{1}, \mathrm{k}_{2}, \mathrm{t}\right)$ have the following limits

$$
\begin{gathered}
\lim _{\mathrm{k}_{2} \rightarrow 0}\left(\frac{\mathrm{e}^{\mathrm{i} 150 \mathrm{k}_{2}}}{\mathrm{i} \mathrm{k}_{2}}-\frac{\mathrm{e}^{\mathrm{i} 50 \mathrm{k}_{2}}}{\mathrm{ik_{2 }}}\right)=100 \\
\lim _{\mathrm{k}_{2} \rightarrow 0}\left(\frac{\sin \left(50 \mathrm{k}_{2}\right)}{\mathrm{k}_{2}}\right)=50 \\
\lim _{\mathrm{k}_{2} \rightarrow 0}\left(\frac{\mathrm{e}^{-\mathrm{i} 50 \mathrm{k}_{2}}}{\mathrm{i} \mathrm{k_{2 }}}-\frac{\mathrm{e}^{-\mathrm{i} 150 \mathrm{k}_{2}}}{\mathrm{i} \mathrm{k} \mathrm{k}_{2}}\right)=100
\end{gathered}
$$

3) As $\mathrm{k}_{1} \rightarrow 0$, then the singular terms of $\bar{\eta}\left(\mathrm{k}_{1}, \mathrm{k}_{2}, \mathrm{t}\right)$ have the following limits

$$
\begin{gathered}
\lim _{k_{1} \rightarrow 0}\left(\frac{1}{i k_{1}}-\frac{e^{-i 50 k_{1}}}{i k_{1}}\right)=50 \\
\lim _{k_{1} \rightarrow 0}\left(\frac{e^{-i k_{1}\left(50+v\left(t-t_{1}\right)\right)}}{i k_{1}}-\frac{e^{-i k_{1}\left(100+v\left(t-t_{1}\right)\right)}}{i k_{1}}\right)=50
\end{gathered}
$$

We investigated the water wave motion in the near and far-field by considering two kinematic in sequence representation of the sea floor faulting, one with vertical uplift motion with time followed by unilateral spreading in $\mathrm{x}$-direction, both with constant velocity v. Clearly, from the mathematical derivation done above, $\eta(\mathrm{x}, \mathrm{y}, \mathrm{t})$ depends continuously on the source $\zeta(\mathrm{x}, \mathrm{y}, \mathrm{t})$. Hence, from the mathematical point of view, this problem is said to be well-posed for modeling the physical processes of the tsunami wave.

\section{Results and Discussion}

We are interest in illustrating the nature of the tsunami build up and propagation during and after the uplift process. In addition, searching for explanations for abnormally large tsunami amplitudes, we demonstrate the waveform amplification resulting from source spreading and wave focusing in the near-field. We first examine the significance of the spreading velocity of the ocean floor uplift by comparing displacement waveforms along the $\mathrm{x}$-axis and in 3-dimendional frame of work for various values of the ratio $\underline{\mathrm{V}}$.

\subsection{Effect of $\frac{v}{v_{t}}$ on Tsunami Waveform}

In this section, we study the focusing and the amplifica- tion of the tsunami amplitude, determined by the velocity of spreading. The results in Figure 4 show that, at the time when the source process is completed and for rapid lateral spreading $\left(\frac{\mathrm{v}}{\mathrm{v}_{\mathrm{t}}} \geq 10\right)$, the displacement of the free surface above the source resembles the displacement of the ocean floor. For velocities of spreading smaller than $v_{t}$ $\left(\frac{\mathrm{v}}{\mathrm{v}_{\mathrm{t}}}<1\right)$, the tsunami amplitudes in the direction of the source propagation become small with high frequencies. As the velocity of the spreading approaches vt, the tsunami waveform has progressively larger amplitude, with high frequency content, in the direction of the slip spreading, Figure 5. These large amplitudes are caused by wave focusing (i.e. during slow earthquakes). It was observed from past tsunami, that slow earthquakes $(0.1<\mathrm{v}<1$ $\mathrm{km} / \mathrm{sec}$ i) may consist of one or several high velocity rupture events, which thus produce the usual train of high frequency waves, with long delays between the successive events, accompanied by the source, which can contribute large amplitude and low-frequency excitation, see [33]. Examples of such slow earthquakes are the June 6, 1960, Chile earthquakes which ruptured as a series of earthquakes for about an hour, [34], and the February 21, 1978, Banda Sea earthquake, [35]. In our case, we concern about studying the amplification of the tsunami amplitude, determined by the velocity of spreading of a single-fault source and it will explain in details later on.

It is difficult to estimate, at present, how often this type of amplification may occur during actual slow submarine process, because of the lack of detailed knowledge about the ground deformations in the source area of past tsunamis. Therefore, we presented here only the basic ideas and illustrate the possible range of amplification factors by means of a realistic curvilinear slip-fault.

The amplification shown in Figure 4 and 5 depends on the spreading velocity $\mathrm{v}$ and the time $\mathrm{t}$ taken to spread the motion over the entire source region. This observation can be verified by comparing these results with the tsunami waveforms obtained by using a simple kinematic source model represented by a sliding Heaviside step function. This case was studied by Todorovska and Trifunac [21]. They considered a square source model characterized by $\mathrm{W}=\mathrm{L}=50 \mathrm{~km}$, with uniform final elevation $\zeta_{0}$, and the velocity of lateral spreading of the ocean floor uplift was constant. We expand the propagation length $\mathrm{L}$ to $150 \mathrm{~km}$ and $\mathrm{W}$ to $100 \mathrm{~km}$ for the Heaviside step function in order to make comparison with the results we obtained. We assumed the beginning of the spreading from $\mathrm{x}=0$ to $\mathrm{x}=150 \mathrm{~km}$ for both cases.

$$
\zeta(\mathrm{x}, \mathrm{y}, \mathrm{t})=\zeta_{0} \mathrm{H}\left(\mathrm{t}-\frac{\mathrm{x}}{\mathrm{v}}\right),(\mathrm{x}, \mathrm{y}) \in\left[(0, \mathrm{~L}) \times\left(\frac{-\mathrm{w}}{2}, \frac{\mathrm{w}}{2}\right)\right]
$$

Applying the Laplace-Fourier transform on $\zeta(\mathrm{x}, \mathrm{y}, \mathrm{t})$, then substituting it in (17) and using the inverse Lap- 

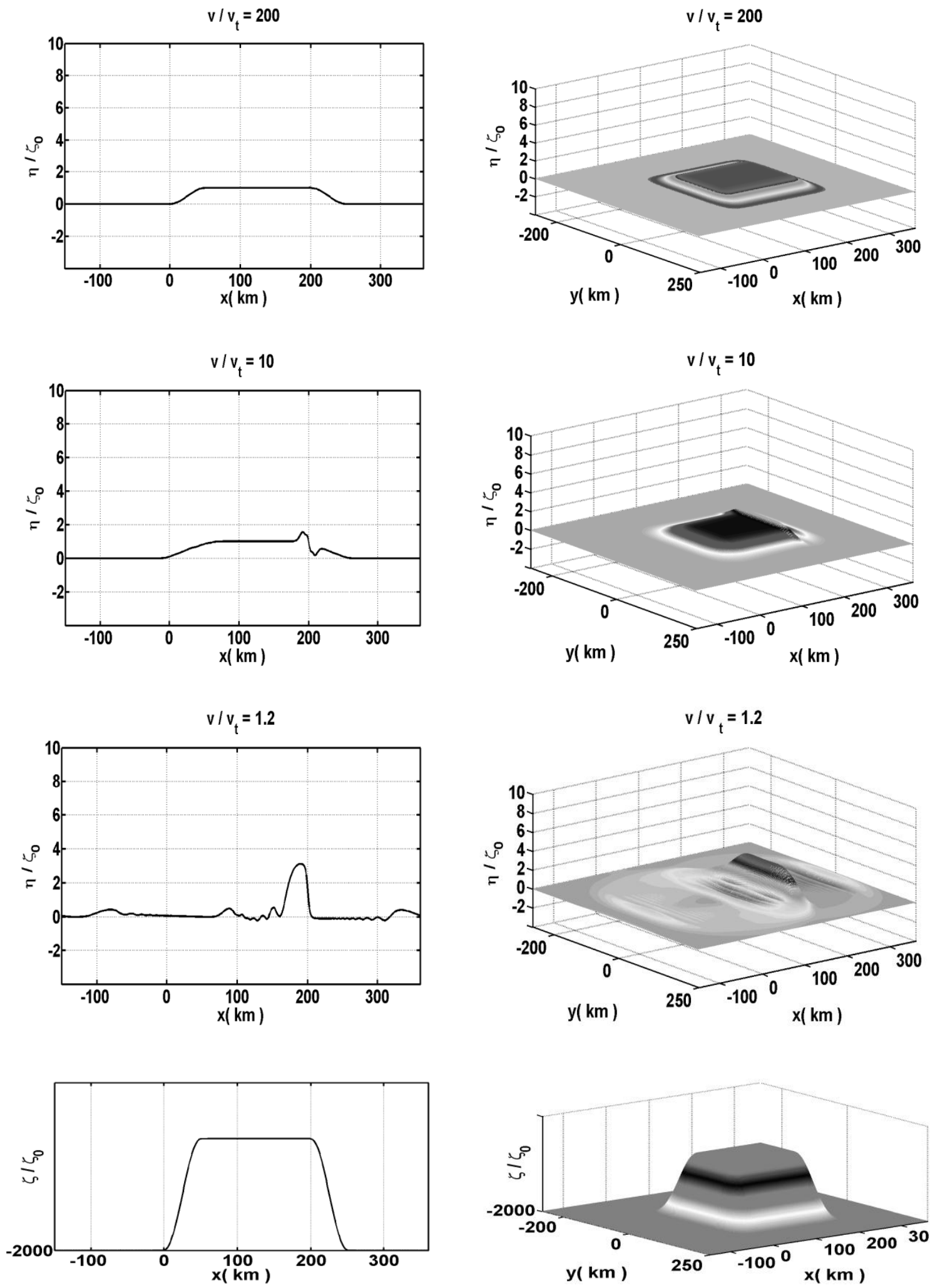

(a)

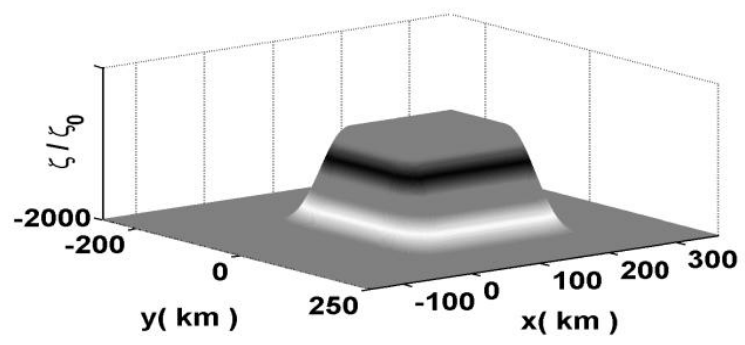

(b)

Figure 4. Dimensionless free-surface deformation $\eta\left(x, y, t^{*}\right) / \zeta_{0}$ for $v / v_{t}>1$ at $h=2 \mathrm{~km}, \mathrm{~L}=150 \mathrm{~km}, \mathrm{~W}=100 \mathrm{~km}, \mathrm{v}_{\mathrm{t}}=0.14$ $\mathrm{km} / \mathrm{sec}$ and $t^{*}=200 / \mathrm{v}$ sec. (a) Side view along the axis of the symmetry at $\mathrm{y}=0$; (b) Three dimensional view. 

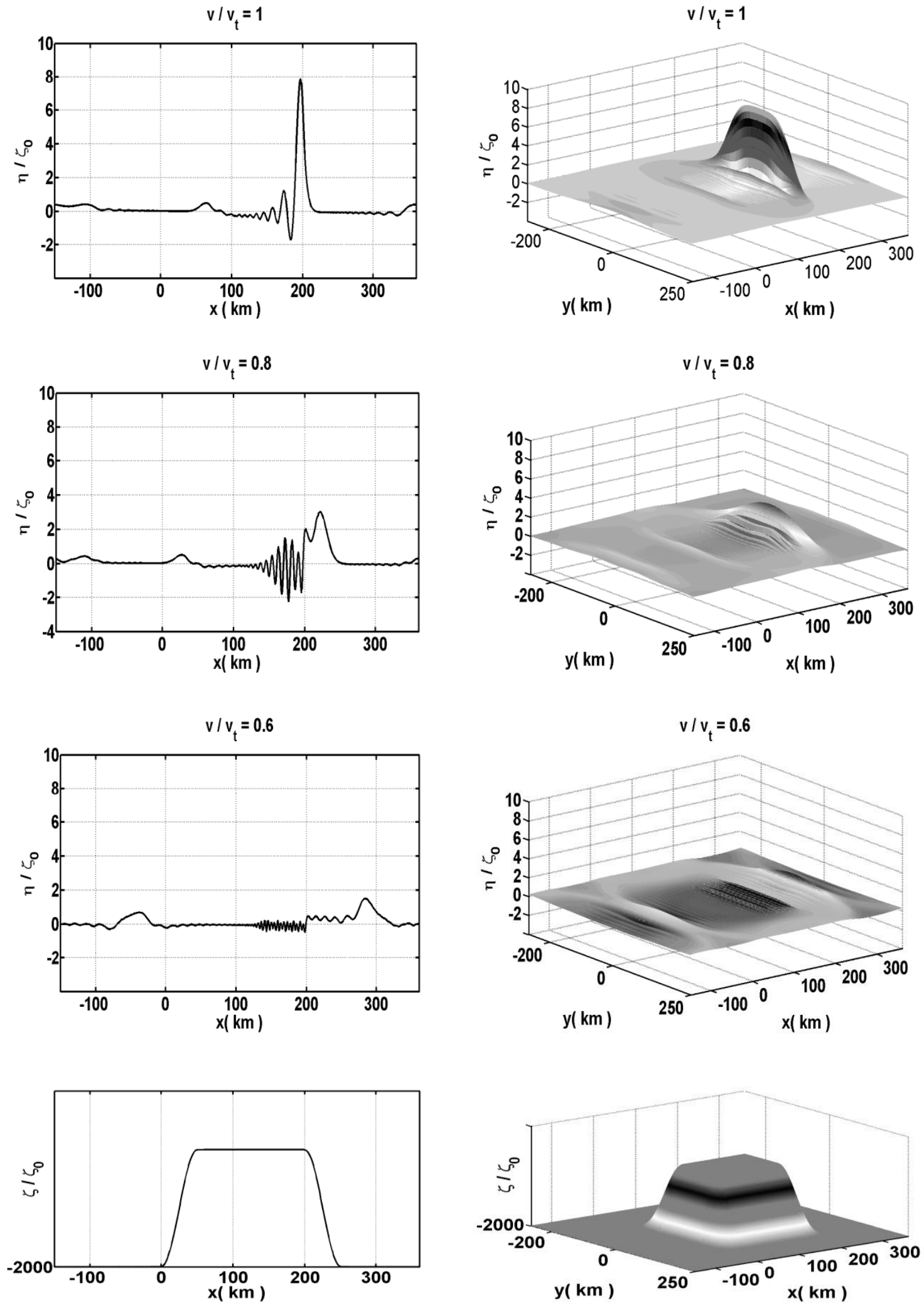

(a)

(b)

Figure 5. Dimensionless free-surface deformation $\eta\left(x, y, t^{*}\right) / \zeta_{0}$ for $v / v_{t} \leq 1$ at $h=2 \mathrm{~km}, L=150 \mathrm{~km}, W=100 \mathrm{~km}, v_{t}=0.14$ $\mathrm{km} / \mathrm{sec}$ and $t^{*}=200 / \mathrm{v}$ sec. (a) Side view along the axis of the symmetry at $\mathrm{y}=0$; (b) Three dimensional view. 
lace transform of $\bar{\eta}\left(\mathrm{k}_{1}, \mathrm{k}_{2}, \mathrm{~s}\right)$ yields for $\mathrm{t} \leq \mathrm{t}^{*}$.

$$
\bar{\eta}\left(\mathrm{k}_{1}, \mathrm{k}_{2}, \mathrm{t}\right)=\left[\frac{2 \sin \left(50 \mathrm{k}_{2}\right)}{\mathrm{k}_{2}}\right]\left[\frac{\zeta_{0} \mathrm{v}}{\cosh (\mathrm{kh})}\left(\frac{1}{\omega^{2}-\left(\mathrm{k}_{1} \mathrm{v}\right)^{2}}\left(\omega \sin \omega \mathrm{t}+\mathrm{ik}_{1} \mathrm{v} \cos \omega \mathrm{t}-\mathrm{ik}_{1} \mathrm{ve}^{-\mathrm{ik}_{1} \mathrm{vt}}\right)\right]\right.
$$

Hence, $\eta(\mathrm{x}, \mathrm{y}, \mathrm{t})$ can be computed using inverse FFT of $\bar{\eta}\left(\mathrm{k}_{1}, \mathrm{k}_{2}, \mathrm{t}\right)$.

The removable singularities in this case are given by the following limits as

1) As $\mathrm{k} \rightarrow 0$, then $\bar{\eta}\left(\mathrm{k}_{1}, \mathrm{k}_{2}, \mathrm{t}\right)$ has the following limit

$$
\lim _{\mathrm{k} \rightarrow 0} \bar{\eta}\left(\mathrm{k}_{1}, \mathrm{k}_{2}, \mathrm{t}\right)= \begin{cases}\zeta_{0} 100 \mathrm{vt} & \mathrm{t} \leq \mathrm{t}^{*} \\ \zeta_{0} 100 \mathrm{vt} & \mathrm{t} \geq \mathrm{t}^{*}\end{cases}
$$

where $\mathrm{t}^{*}=\frac{150}{\mathrm{v}}$

2) As $\mathrm{k}_{2} \rightarrow 0$, then the singular term of $\bar{\eta}\left(\mathrm{k}_{1}, \mathrm{k}_{2}, \mathrm{t}\right)$ has the following limit

$$
\lim _{\mathrm{k}_{2} \rightarrow 0}\left(\frac{2 \sin \left(50 \mathrm{k}_{2}\right)}{\mathrm{k}_{2}}\right)=100
$$

No singular terms needed to remove as $\mathrm{k}_{1} \rightarrow 0$.
Table 2 shows the comparison for the peak tsunami amplitude $\eta_{\max } / \zeta_{0}$ at certain values of the spreading velocity, for the curvilinear slip-fault source and the simple rectangular-slide with $\mathrm{L}=150 \mathrm{~km}$ and $\mathrm{W}=100 \mathrm{~km}$.

Figure 6 illustrates the tsunami waveforms generated by the slip-fault model and the rectangular-slide model with $\mathrm{L}=150 \mathrm{~km}$ and $\mathrm{W}=100 \mathrm{~km}$ when the maximum amplitude amplification occurs at $\mathrm{v}=\mathrm{v}_{\mathrm{t}}$.

It can be observed from Table 2 that, at certain value of $\mathrm{v}$, the waveform generated by the slip-fault model and the rectangular-slide has little different peak amplitudes. This happens as a result of wave focusing covers a wider area (curvilinear region) above the source which needed more time for amplification. For example, at $\mathrm{v}=\mathrm{v}_{\mathrm{t}}$, where the time for the slip-fault takes $23.8 \mathrm{~min}$ to complete the entire source region with peak amplitude equal to $7.906 \mathrm{~m}$, while for the rectangular-slide the time duration takes $17.8 \mathrm{~min}$ to reach a maximum amplitude equal to $7.524 \mathrm{~m}$.

Table 2. Peak tsunami amplification for the curvilinear slip-fault model and the rectangular-slide at different values of the spreading velocity $v$.

\begin{tabular}{lll}
\hline spreading velocity $\mathbf{v}(\mathrm{km} / \mathrm{sec})$ & $\begin{array}{l}\boldsymbol{\eta}_{\max } / \boldsymbol{\zeta}_{\mathbf{0}} \\
\text { (slip-fault) }\end{array}$ & $\begin{array}{l}\boldsymbol{\eta}_{\max } / \boldsymbol{\zeta}_{\mathbf{0}} \\
\text { (rectangular-slide) }\end{array}$ \\
\hline $\mathrm{v}=200 \mathrm{vt}=28$ & 1 & 1 \\
$\mathrm{v}=20 \mathrm{vt}=2.8$ & 1.481 & 1.003 \\
$\mathrm{v}=10 \mathrm{vt}=1.4$ & 1.575 & 1.010 \\
$\mathrm{v}=5 \mathrm{vt}=0.7$ & 1.615 & 1.042 \\
$\mathrm{v}=2 \mathrm{vt}=0.28$ & 1.880 & 1.333 \\
$\mathrm{v}=1.2 \mathrm{vt}=0.168$ & 3.145 & 3.204 \\
$\mathrm{v}=\mathrm{vt}=0.14$ & 7.906 & 7.524 \\
$\mathrm{v}=0.8 \mathrm{vt}=0.112$ & 3.014 & 1.958 \\
$\mathrm{v}=0.6 \mathrm{vt}=0.084$ & 1.498 & 0.569 \\
\hline
\end{tabular}
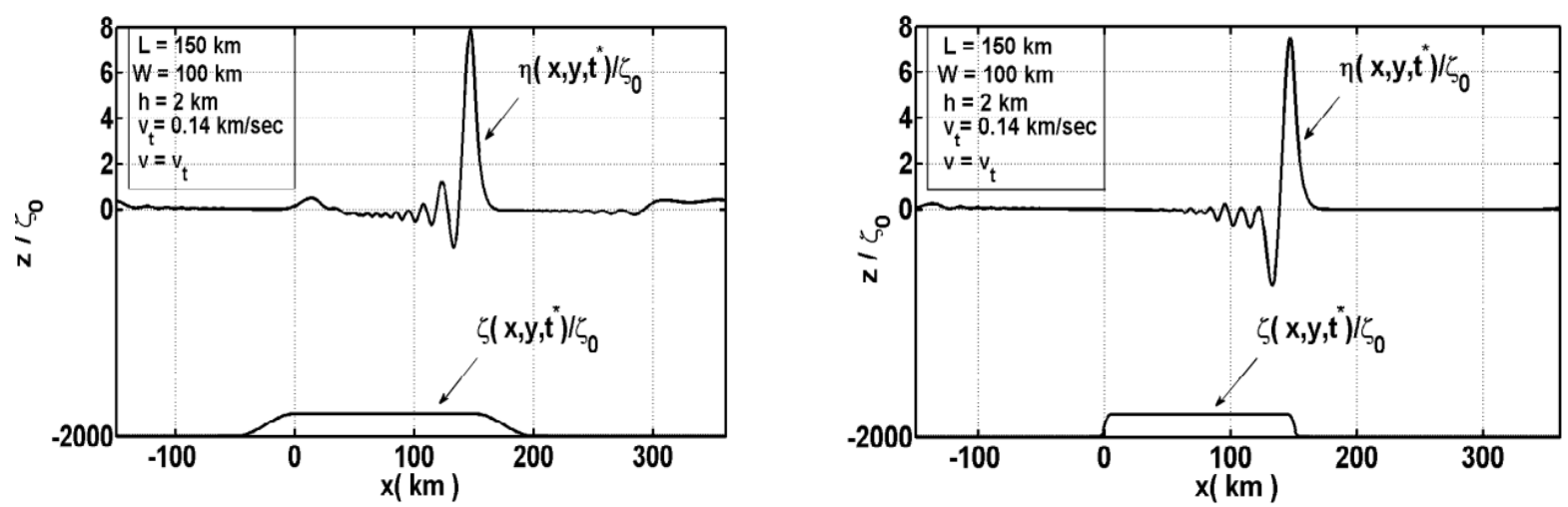

Figure 6. Maximum tsunami amplitude at spreading velocity $v^{=} v_{t}$, generated by. (a) Slip-fault source; (b) Rectangular-slide source. 
For $\mathrm{v}>\mathrm{v}_{\mathrm{t}}$, the maximum amplitudes calculated are small comparable to the peak amplitude at $\mathrm{v}=\mathrm{v}_{\mathrm{t}}$ as shown in Figure 4 and Table 2. These values are logic due to rapid movements of the bed $\left(v=10 v_{t}\right.$ and $\left.v=200 v_{t}\right)$, which progressively approximated the shape of the bed deformation. This phenomenon agrees with the physical process of tsunami wave produced by earthquakes. For $\mathrm{v}=0.6 \mathrm{v}_{\mathrm{t}}$ and $\mathrm{v}=0.8 \mathrm{v}_{\mathrm{t}}$ where the tsunami is faster than the uplift, the initial wave escapes ahead of the currently uplifted water and the amplitudes of the tsunami waves above the source become smaller with higher frequency content continues and hence no amplification will occur as shown in Figure 5(a). At the instant the source motion has been stopped, it may cover larger area than the area of the source due to dispersion as demonstrated in Figure 5(b).

\subsection{Tsunami Generation and Propaga- tion-Evolution in Time}

We study the effects of variations of the uplift as a function of time in the slowly uplift faulting and the spreading slip fault sources in generating and propagating of tsunamis above and away from the sources. The generation of tsunamis by vertical displacements of the ocean floor depends on the characteristic size (length L and width $\mathrm{W}$ ) of the displaced area and on the time $\mathrm{t}$, taken to spread the motion over the entire source region. The ratio $\mathrm{L} / \mathrm{t}$ then defines the average spreading (or fault rupture) velocity $\mathrm{v}$, assuming unilateral spreading of the slip-fault along length L. We choose the velocity of the sea floor spreads similar to the long wave tsunami velocity $\mathrm{v}_{\mathrm{t}}$ (i.e. maximum amplification).We illustrate the final uplifted area for the slowly uplift faulting by $\mathrm{L}=100 \mathrm{~km}$ and $\mathrm{W}=100 \mathrm{~km}$, and for the slip-fault by length of propagation $\mathrm{L}=150 \mathrm{~km}$ and $\mathrm{W}=100 \mathrm{~km}$ with constant spreading velocity v. Figures 7 and 9 a show the tsunami generated waveforms at times $t=0.4 t^{*}, 0.6 t^{*}, 0.8 t^{*}, t^{*}$. It is seen how the amplitude of the wave builds up progressively as $t$ increases. Figures 8 and 9(b) illustrate the propagation process of the tsunami wave away from the source for times between $\mathrm{t}=2 \mathrm{t}^{*}$ and $\mathrm{t}=3.5 \mathrm{t}^{*}$. Table 3 represents the determined values $\eta_{\max } / \zeta_{0}$ by the slip-fault source during the times $\mathrm{t}=0.2 \mathrm{t}^{*}, 0.4 \mathrm{t}^{*}, 0.6 \mathrm{t}^{*}, 0.8 \mathrm{t}^{*}, \mathrm{t}^{*}$. At $\mathrm{v}=\mathrm{v}_{\mathrm{t}}$, the wave will be focusing and the amplification may occurs above the spreading edge of the slip as shown in Figure 9a. This amplification occurs above the source progressively, as the source evolves, by adding uplifted fluid to the fluid displaced previously by uplifts of preceding source segments. This explains why the amplification is larger for wider area of uplift source, than for small source area.

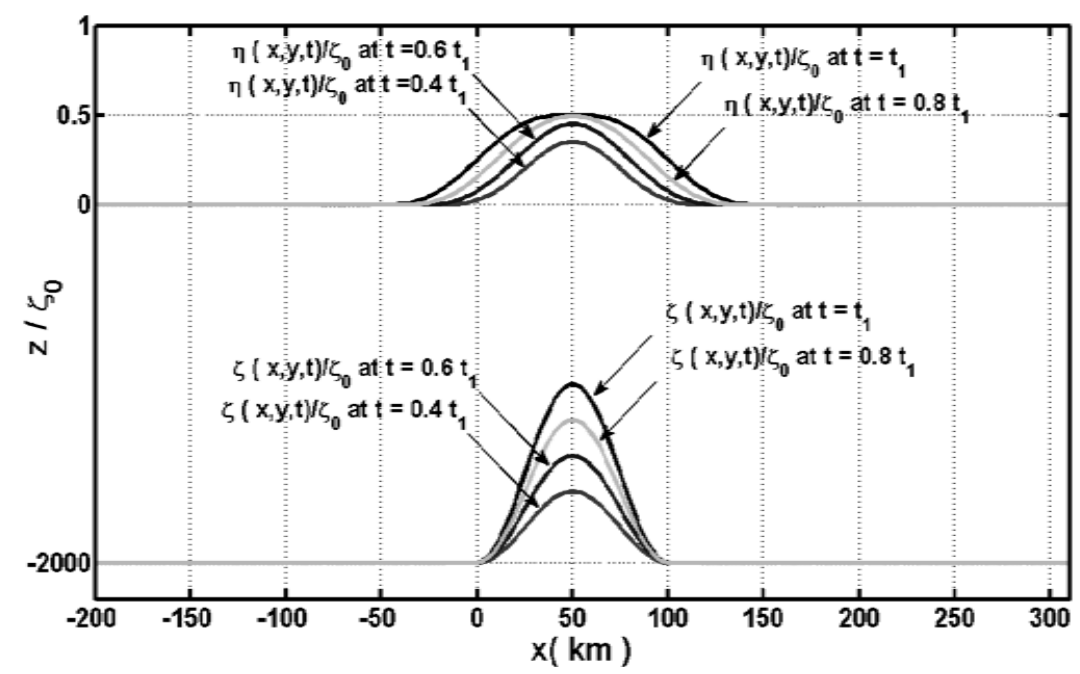

Figure7. Tsunami generated waveforms by a curvilinear uplift source with characteristic size $L=100 \mathrm{~km}$ and $\mathrm{W}=100 \mathrm{~km}$ at different uplift times $t$ at $v=v_{t}$ and $t^{*}=t_{1}=\frac{50}{v}$ sec.

Table 3.Values of $\eta_{\max }(x, y, t) / \zeta_{0}$ at different rise time $t$ and at $v=v_{t}$.

\begin{tabular}{ll}
\hline Rise time t & $\boldsymbol{\eta}_{\max } / \zeta_{0}$ \\
\hline $\mathrm{t}=0.2 \mathrm{t}^{*}$ & 3.065 \\
$\mathrm{t}=0.4 \mathrm{t}^{*}$ & 4.385 \\
$\mathrm{t}=0.6 \mathrm{t}^{*}$ & 5.637 \\
$\mathrm{t}=0.8 \mathrm{t}^{*}$ & 6.800 \\
$\mathrm{t}=\mathrm{t}^{*}$ & 7.906 \\
\hline
\end{tabular}



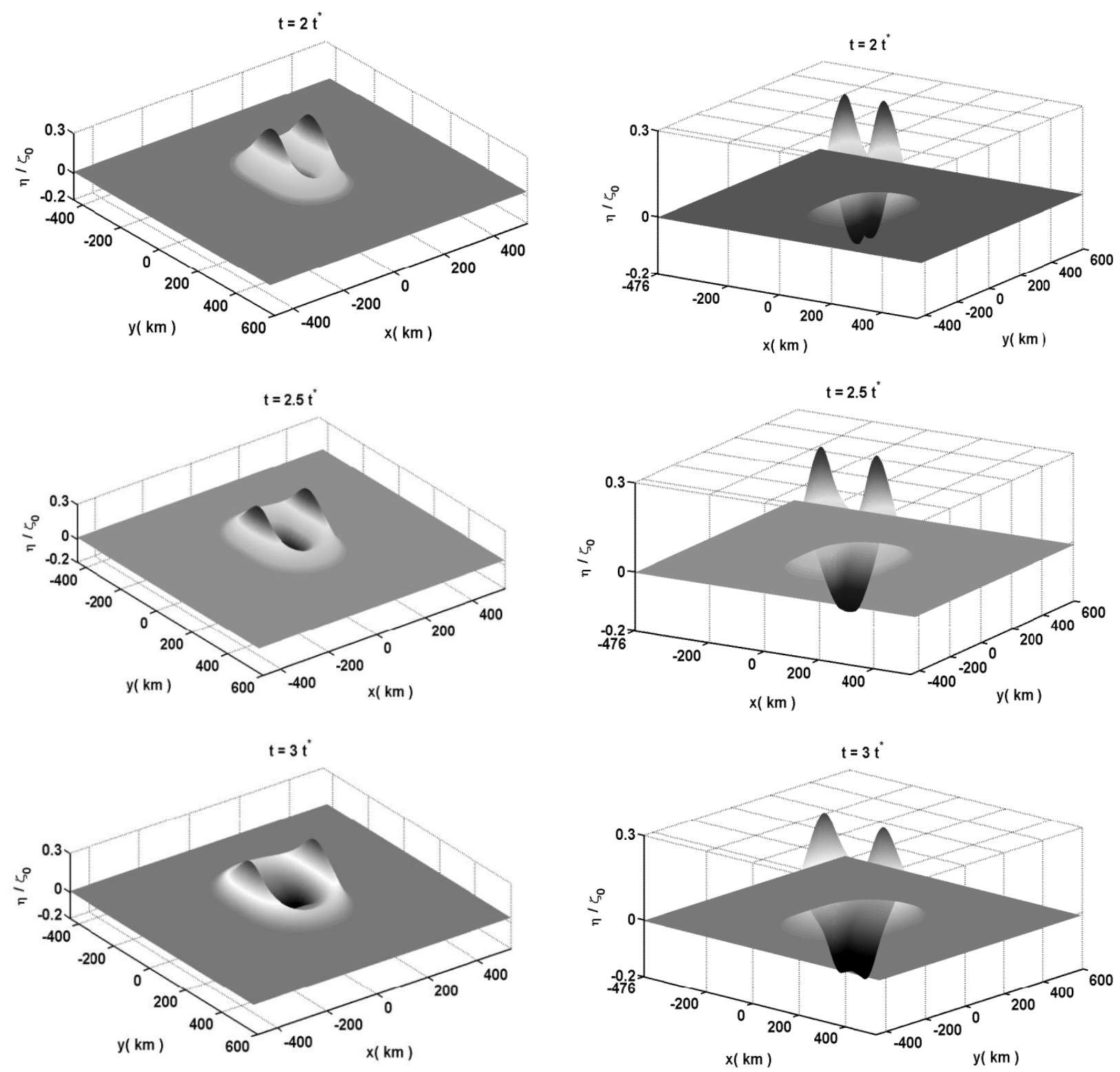

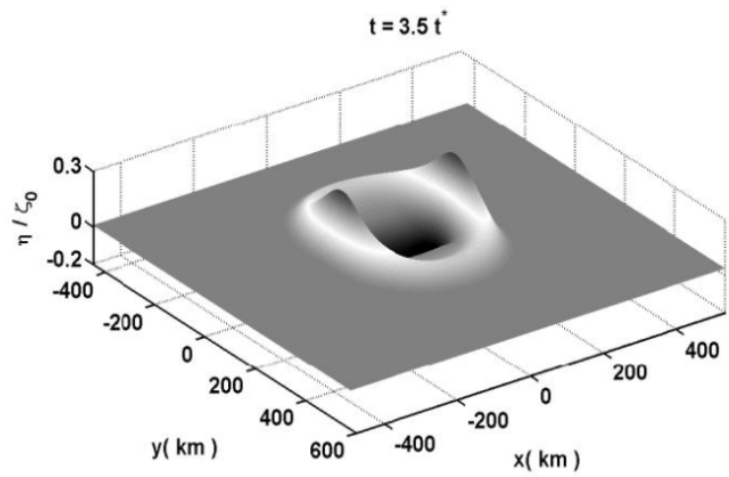

(a)

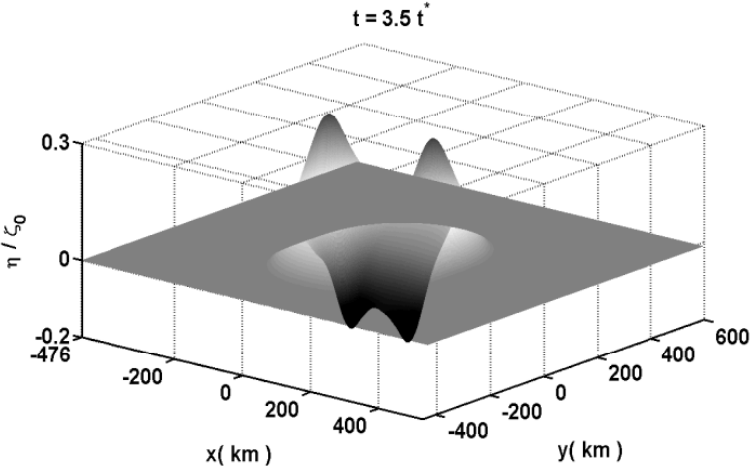

(b)

Figure 8. Tsunami propagated waveforms following the curvilinear uplift source with characteristic size $\mathbf{L}=100 \mathrm{~km}$ and $\mathrm{W}=100 \mathrm{~km}$ at different rise times $\mathrm{t}$ and $\mathrm{t}^{*}=\frac{50}{\mathrm{v}}$ sec. (a) Top view; (b) Bottom view. 

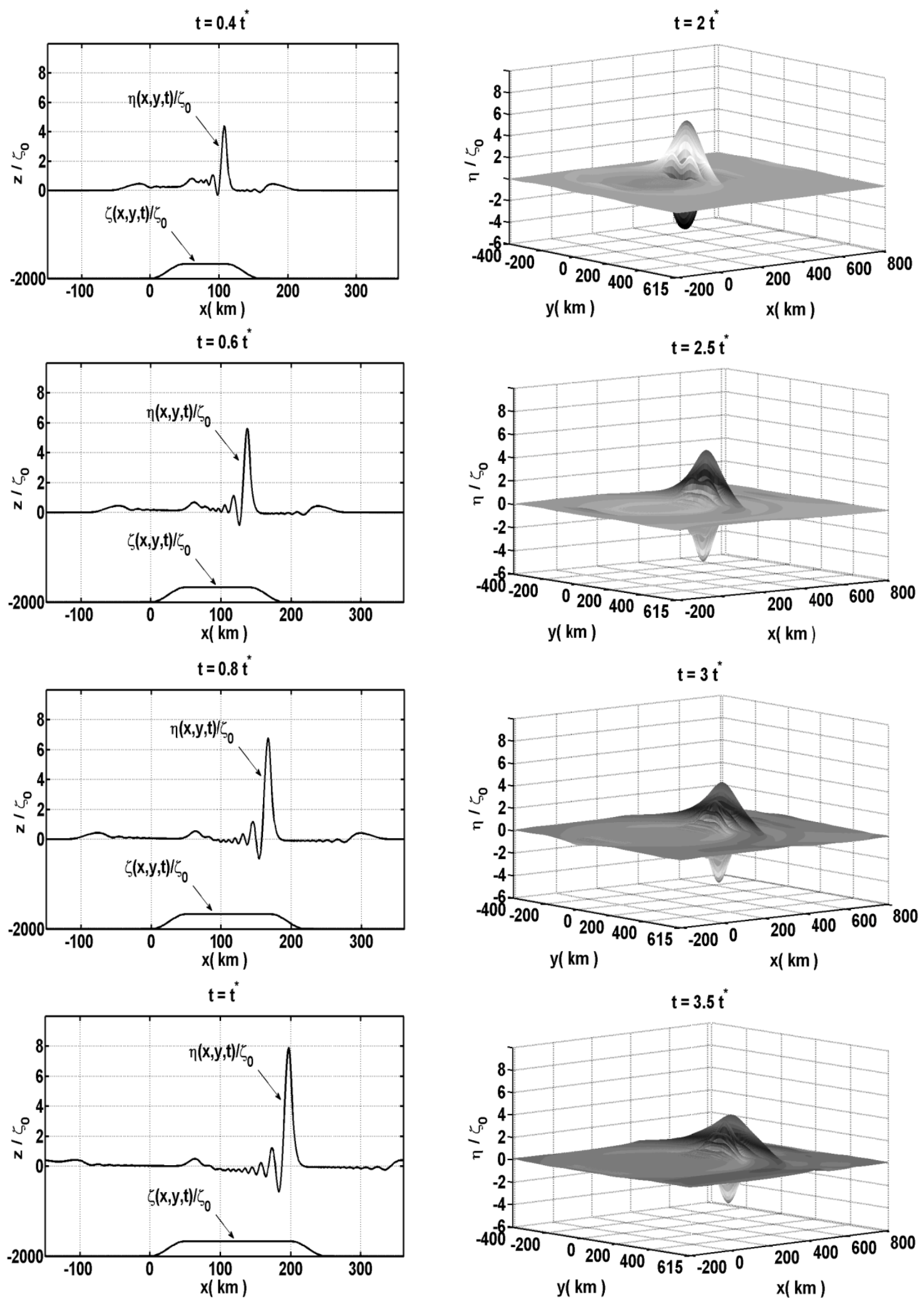

(a)

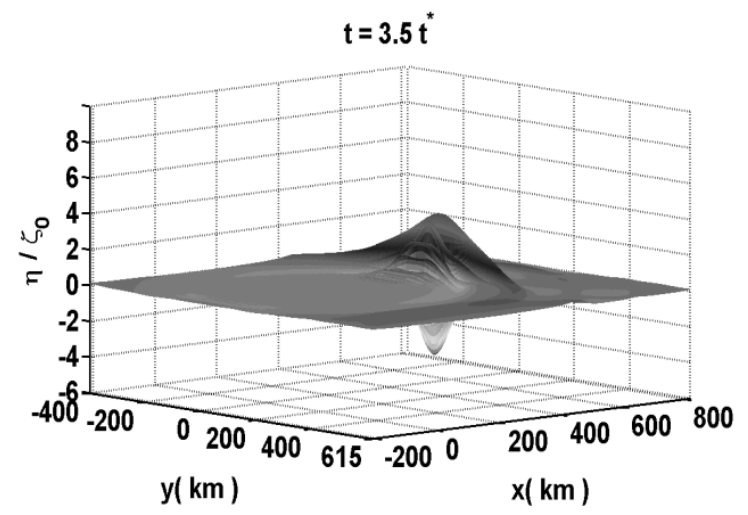

(b)

Figure 9. Tsunami waveform by the slip-fault source with $v=v_{t}, L=150 \mathrm{~km}$ and $W=100 \mathrm{~km}$ at different rise time $t$ where $t^{*}=\frac{200}{v}$. (a) Side view of the tsunami generated wave at $y=0 ;$ (b) Three dimensional view of the tsunami propagation. 
For very rapid movements of the bed, the water surface displacement initially approximated the shape of the bed deformation and then divided into two wave trains propagating in opposite directions. The maximum amplitude of the largest wave leaving the generation region for these bed motions was found never to exceed one half of the maximum bed displacement. For slower motions of the bed like the case we study, the maximum wave amplitudes decreased [31]. Similar features relating to the maximum amplitudes of waves propagating from the generation region in a three-dimensional fluid domain were discussed by Nakamura [10], Momoi [11] and Kajiura [12]. The results shown in Figure7 demonstrated the water surface displacement generated by slow motions of the bed. The waveforms approximated the shape of the bed deformation with smaller amplitude than the sea bed which then divided into two wave trains propagating in opposite directions as shown in Figure 8. The maximum amplitude of the largest wave leaving the generation region for this bed motions was one half of the maximum initial wave displacement. This agrees with the typical phenomena of past tsunami wave generated by slowly earthquakes.

In case of the curvilinear slip-fault, we assume the waveform was initiated by end stage of the uplift source, and then propagated in the $\mathrm{x}$-direction with constant velocity v. It is seen from Figure 9a, how the amplitude of the wave builds up as progressively more water is lifted below the leading wave depending on its variation in time and the space in the source area. The parameter that governs the amplification of the near-field water waves by focusing is the ratio of the spreading length $L$ over the water depth, L/h, as shown in Figure 9a and Table 4. As the spreading length in the slip-fault increases, the amplitude of the tsunami wave becomes higher. As the wave propagates, the wave height decreases and the slope of the front of the wave becomes smaller, causing a train of small waves forms behind the main wave, as shown in Figure 9b. The maximum wave amplitude decreases with time, due to the geometric spreading and also due to the dispersion. At $\mathrm{t}=3.5 \mathrm{t}^{*}$, the wave front is at about $\mathrm{x}=567 \mathrm{~km}$ and $\eta / \zeta_{0}$ decreases to 3.313.This happens because the amplification of the waveforms in the far-field does not depend on the source velocity, but only on the volume of the displaced water by the source process which become an important factor in modeling the generation of tsunami. This was clear from the singular points removed for the two source models, where the finite limits of the free surface depends on the characteristic volume of the source models.

Table 3 shows the variation in the amplification factor $\eta_{\max } / \zeta_{0}$ for various values of the rise time $\mathrm{t}$ at $\mathrm{h}=2 \mathrm{~km}$ and $\mathrm{t}=\mathrm{t}^{*}=\mathrm{L} / \mathrm{v}$. It can observe that, the peak amplitude increases as the rise time increases. This is due to the amplification mainly depends on the length of propagation L.

Table 4 shows the variation in the amplification factor $\eta_{\max } / \zeta_{0}$ for various values of the propagated uplift length $\mathrm{L}$ and width $\mathrm{W}$ at $\mathrm{h}=2 \mathrm{~km}$ and $\mathrm{t}=\mathrm{t}_{1}+\mathrm{L} / \mathrm{v}$. Note that at $\mathrm{L}=$ 0 , no propagation occurs and the waveform takes initially the shape and amplitude of the curvilinear uplift fault (i.e. $\left.\eta_{\max } / \zeta_{0}=1\right)$. It is seen from Table 4 that for $\mathrm{L} / \mathrm{h}$ between 0 and $500, \eta_{\max } / \zeta_{0}$ varies from 1 to 27.82 . It can be observed that the amplification increases with the increase in $\mathrm{L} / \mathrm{h}(0-500)$ and with the increase in $\mathrm{W} / \mathrm{L}(0.25-5)$. The increase in the propagation length produces larger amplification than the increase in the width. This happens from the assumption that the source model propagated instantaneously in the y-direction. This leaves us to study the generation of tsunami by spreading curvilinear slides and slumps in two directions in Future work.

Figure 10 represents the values of the normalized peak wave amplitude $\eta_{\max } / \zeta_{0}$ at $\mathrm{v}=\mathrm{v}_{\mathrm{t}}$ and $\mathrm{h}=2 \mathrm{~km}$ for different values for W/L obtained from Table 4.

Table 5 presents the effect of the water depth $h$ on the amplification factor $\eta_{\max } / \zeta_{0}$ for various values of the ratios $\mathrm{W} / \mathrm{L}$ and at constant propagation $\mathrm{L}=150 \mathrm{~km}$. It is seen that for $\mathrm{h}$ between 0.5 and $6 \mathrm{~km}, \eta_{\max } / \zeta_{0}$ is varies from 19.77 to 3.565 . The values determined in Table 5 shows that the maximum amplitude amplification increases with the decrease in h. This phenomenon happens because the speed of the tsunami is related to the water depth which produces small wavelength as the velocity decreases and hence the height of the wave grows as the change of total energy of the tsunami remains constant. Mathematically, wave energy is proportional to both the length of the wave and the height squared. Therefore, if the energy remains constant and

Table 4. Values of $\eta_{\max } / \zeta_{0}$ at $v=v t$ and $h=2 \mathrm{~km}$ with various values of $L$ and $W$ and $t=t_{1}+L / v$.

\begin{tabular}{lccccc}
\hline $\begin{array}{c}\mathbf{L} / \mathbf{h} \\
(\mathbf{h}=\mathbf{2} \mathbf{~ k m})\end{array}$ & $\mathbf{0 . 2 5}$ & $\mathbf{0 . 5}$ & $\mathbf{W} / \mathbf{L}$ & $\mathbf{2}$ & $\mathbf{5}$ \\
\hline $\mathbf{0}$ & 1.0000 & 1.0000 & 1.0000 & 1.0000 & 1.0000 \\
$\mathbf{5}$ & 1.9190 & 1.9230 & 1.9290 & 1.9320 & 1.9330 \\
$\mathbf{1 0}$ & 2.5410 & 2.5550 & 2.5720 & 2.5840 & 2.5840 \\
$\mathbf{2 5}$ & 3.7820 & 3.8250 & 3.8780 & 3.9260 & 3.9270 \\
$\mathbf{5 0}$ & 5.9160 & 5.9920 & 6.0330 & 6.1210 & 6.1220 \\
$\mathbf{1 0 0}$ & 9.3310 & 9.5360 & 9.5390 & 9.5610 & 9.5940 \\
$\mathbf{2 5 0}$ & 16.940 & 17.500 & 17.530 & 17.540 & 17.540 \\
$\mathbf{5 0 0}$ & 27.360 & 27.780 & 27.790 & 27.820 & 27.820 \\
\hline
\end{tabular}


Table 5. Values of $\eta_{\max } / \zeta_{0}$ at $v=g h, L=150 \mathrm{~km}$ and $t^{*}=200 / \mathrm{v}$ for various values of the $W / L$.

\begin{tabular}{lccccc}
\hline $\mathbf{h}(\mathbf{~ k m ~ )}$ & $\mathbf{0 . 2 5}$ & $\mathbf{0 . 5}$ & $\mathbf{W} / \mathbf{L}$ & $\mathbf{2}$ & $\mathbf{5}$ \\
\hline $\mathbf{0 . 5}$ & 19.690 & 19.730 & 19.730 & 19.770 & 19.770 \\
$\mathbf{1}$ & 12.410 & 12.510 & 12.510 & 12.560 & 12.560 \\
$\mathbf{2}$ & 7.7610 & 7.8990 & 7.9100 & 7.9730 & 7.9740 \\
$\mathbf{3}$ & 5.8600 & 6.0190 & 6.0430 & 6.1160 & 6.1180 \\
$\mathbf{4}$ & 4.7800 & 4.9490 & 4.9880 & 5.0680 & 5.0710 \\
$\mathbf{5}$ & 4.0670 & 4.2400 & 4.2920 & 4.3790 & 4.3820 \\
$\mathbf{6}$ & 3.5650 & 3.7290 & 3.7920 & 3.8840 & 3.8880 \\
\hline
\end{tabular}

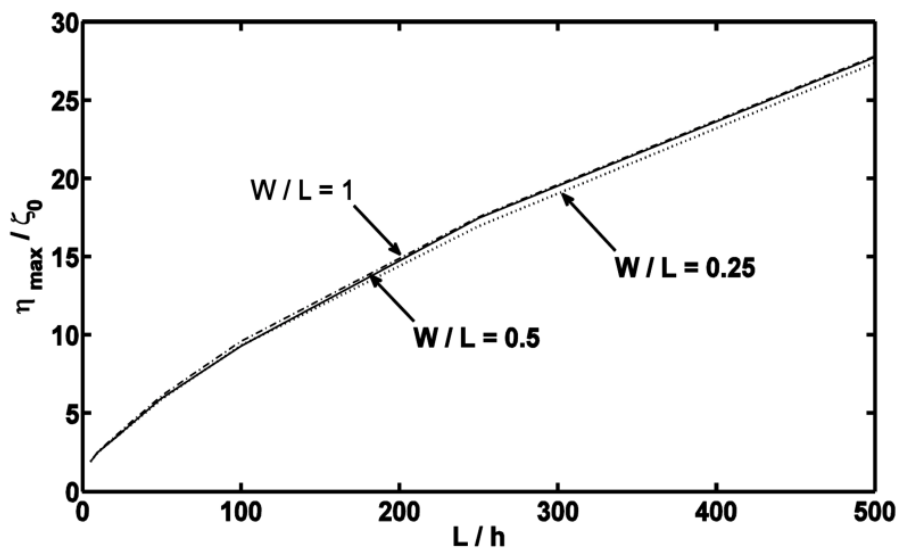

Figure 10. The normalized peak wave amplitude $\eta_{\max } / \zeta_{0}$ versus the dimensionless parameter $L / h$ for $v=v_{t}, W / L \geq 0.25$ and $h$ $=\mathbf{2} \mathbf{k m}$.

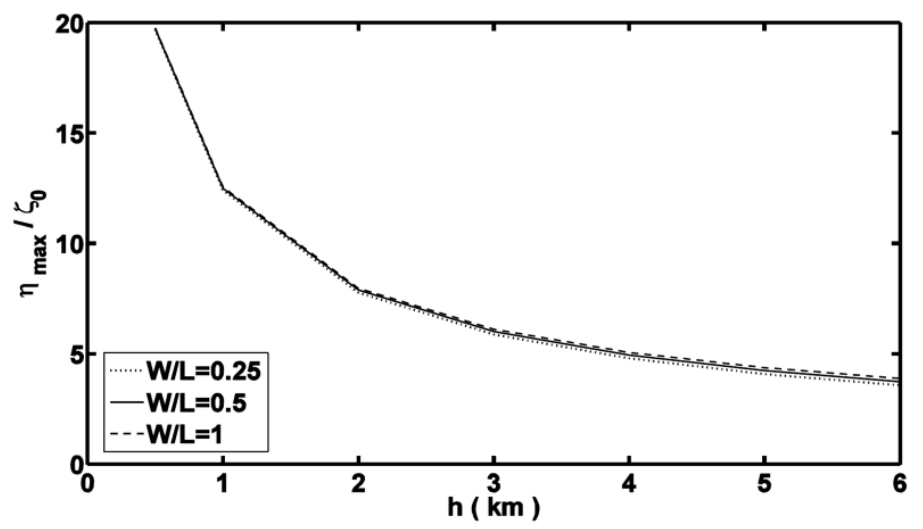

Figure 11. The normalized peak wave amplitude $\eta_{\max } / \zeta_{0}$ versus the water depth $\mathrm{h}$ for $\mathrm{v}=\mathrm{vt}, \mathrm{W} / \mathrm{L} \geq 0.25$ and propagation length $L=150 \mathrm{~km}$.

the wavelength decreases, then the height must increase. These results agree with the aspect obtained by Hayir [36] who determined the effects of ocean depth on tsunami amplitudes for simple kinematic source models.

Figure 11 represents the values of the normalized peak wave amplitude $\eta_{\max } / \zeta_{0}$ for different values of water depth $\mathrm{h}$ and the ratios $\mathrm{W} / \mathrm{L}$ at constant propagation $\mathrm{L}$ $=150 \mathrm{~km}$.

\section{Conclusions}

In this paper, we presented a review of the main physical characteristics of a realistic tsunami sources. We consi- dered two curvilinear source models represented by a slowly uplift faulting followed by a slip-fault model. We studied the effect of the source propagation and wave focusing on the amplitudes of the tsunami generated and propagated by the dynamic source models considered.The results showed that the amplitude amplification of up to 8 order of magnitude occurs in the direction of source propagation when the velocity of the source is close to the long period tsunami velocity. This means that amplification occurs above the source, progressively, as the source evolves, by adding uplifted fluid to the fluid displaced previously by uplifts of preceding source segments. This amplification depends on the characteristic 
size of the displaced area and the time it takes to spread the motion over the entire source region. It is observed that near the source, the wave has large amplitude with short wavelength pulse, while as the tsunami further departed away from the source the amplitude of this pulse decreased due to dispersion. This happens because in the far-field the peak tsunami amplitude does not depend on the source velocity, but only on the volume of the displaced water by the source process. The results show that, the largest peak of the tsunami amplitude at time $t^{*}$ occurs when $v=v t$ due to wave focusing. It is seen that for source model we considered that spread rapidly $\left(\frac{\mathrm{V}}{\mathrm{v}_{\mathrm{t}}} \geq 10\right)$, the displacement of the free surface resembles the displacement of the ocean floor at time $t^{*}$ (i.e., $\left.\frac{\eta}{\zeta_{0}} \approx 1\right)$. For $\left(\frac{\mathrm{v}}{\mathrm{v}_{\mathrm{t}}}<1\right)$, the peak amplitude decreases due to dispersion with the present of high frequency contents in the wave. These results are in complete agreement with the aspect of the tsunami generated by a slowly spreading uplift of the ocean bottom presented by Todorovsk \& Trifunac [21] who considered a very simple kinematic source model. From this observation, we numerically analyzed the dependence of the peak amplification of the tsunami waveforms by changing the length of propagation, the width of the source and the water depth. It was found that the maximum amplitude amplification is proportion to the propagation length and the width of the source model and inversely proportional with the water depth. The presented analysis suggests that some abnormally large tsunamis could be explained in part by a slowly spreading uplift of the sea floor. Our results should help to enable quantitative tsunami forecasts and warnings based on recoverable seismic data and to increase the possibilities for the use of tsunami data to study earthquakes, particularly historical events for which adequate seismic data do not exist. The estimated near-field tsunami generated under the effect of variable velocity of curvilinear slides and slumps spreading in two directions are underway.

\section{References}

[1] A. Ben-Menahem and M. Rosenman, "Amplitude Patterns of Tsunami Waves from Submarine Earthquakes," Journal of Geophysical Research, Vol. 77, No. 17, 1972, pp. 3097-3128.

[2] E. O. Tuck and L. S. Hwang, "Long Wave Generation on a Sloping Beach," Journal of Fluid Mechanics, Vol. 51, No. 3, pp. 449-461, 1972.

[3] C. E. Synolakis and E. N. Bernard, "Tsunami Science Be-fore and Beyond Boxing Day 2004 Phil.," Philosophical Transactions of the Royal Society of London A, Vol. 364, No. 1845, 2006, pp. 2231-2265.
[4] J. R. Houston and A. W. Garcia, "Type 16 Flood Insurance Study," Usace Waterways Experiment Station Report, No. H-74-3, Vicksburg, 1974.

[5] S. Tinti and E. Bortolucci, "Analytical Investigation on Tsunamis Generated by Submarine Slides," Annali Di Geofisica, Vol. 43, No. 3, 2000, pp. 519-536.

[6] D. Dutykh, F. Dias and Y. Kervella, "Linear Theory of Wave Generation by a Moving Bottom," Comptes Rendus Mathematique, Vol. 343, No. 7, 2006, pp. 499-504.

[7] R. Takahasi and T. Hatori, "A Model Experiment on the Tsunami Generation from a Bottom Deformation Area of Elliptic Shape," Bulletin Earthquake Research Institute, Tokyo University, Vol. 40, 1962, pp. 873-883.

[8] R. Takahasi, "On Some Model Experiments on Tsunami Generation," International Union of Geodesy and Geophysics, Vol. 24, 1963, pp. 235-248.

[9] Y. Okada, "Surface Deformation Due to Shear and Tensile Faults in a Half Space," Bulletin of the Seismological Society of America, Vol. 75, No. 4, 1985, pp. 1135-1154.

[10] K. Nakamura, "On the Waves Caused by the Deformation of the Bottom of the Sea I.," Science Reports of the Tohoku University, Vol. 5, 1953, pp. 167-176.

[11] T. Momoi, "Tsunami in the Vicinity of a Wave Origin," Bulletin Earthquake Research Institute, Tokyo University, Vol. 42, 1964, pp. 133-146.

[12] K. Kajiura, "Leading Wave of a Tsunami," Bulletin Earthquake Research Institute, Tokyo University, Vol.41, 1963, pp. 535-571.

[13] J. B. Keller, "Tsunamis: Water Waves Produced by Earthquakes," International Union of Geodesy and Geophysics, Vol. 24, 1963, pp. 150-166.

[14] Y. Kervella, D. Dutykh and F. Dias, "Comparison between Three-Dimensional Linear and Nonlinear Tsunami Generation Models," Theoretical and Computational Fluid Dynamics, Vol. 21, No. 4, 2007, pp. 245-269.

[15] M. Villeneuve, "Nonlinear, Dispersive, Shallow-Water Waves Developed by a Moving Bed," Journal of Hydraulic Research, Vol. 31, No. 2, 1993, pp. 249-266.

[16] P. L. Liu and J. A. Liggett, "Applications of Boundary Element Methods to Problems of Water Waves," In P. K. Banerjee and R. P. Shaw Eds., Developments in Boundary Element Methods, 2nd Edition, Applied Science Publishers, England, Chapter 3, 1983, pp. 37-67.

[17] J. L. Bona, W. G. Pritchard and L. R. Scott, "An Evaluation of a Model Equation for Water Waves," Philosophical Transactions of the Royal Society of London A, Vol. 302, No. 1471, 1981, pp. 457-510.

[18] M. S. Abou-Dina and F. M. Hassan, "Generation and Propagation of Nonlinear Tsunamis in Shallow Water by a Moving Topography," Applied Mathematics and Computation, Vol. 177, No. 2, 2006, pp. 785-806.

[19] F. M. Hassan, "Boundary Integral Method Applied to the Propagation of Non-Linear Gravity Waves Generated by a Moving Bottom," Applied Mathematical Modeling, Vol. 33, No. 1, 2009, pp. 451-466. 
[20] N. Zahibo, E. Pelinovsky, T. Talipova, A. Kozelkov and A. Kurkin, "Analytical and Numerical Study of Nonlinear Effects at Tsunami Modeling," Applied Mathematics and Computation, Vol. 174, No. 2, 2006, pp. 795-809.

[21] M. I. Todorovsk and M. D. Trifunac, "Generation of Tsunamis by a Slowly Spreading Uplift of the Sea Floor," Soil Dynamics and Earthquake Engineering, Vol. 21, No. 2, 2001, pp. 151-167.

[22] M. D. Trifunac and M. I. Todorovska, "A Note on Differences in Tsunami Source Parameters for Submarine Slides and Earthquakes," Soil Dynamics and Earthquake Engineering, Vol. 22, No. 2, 2002, pp. 143-155.

[23] M. I. Todorovsk, M. D. Trifunac and A. Hayir, "A Note on Tsunami Amplitudes above Submarine Slides and Slumps," Soil Dynamics and Earthquake Engineering, Vol. 22, No. 2, 2002, pp. 129-141.

[24] M. D. Trifunac, A. Hayir and M. I. Todorovska, "A Note on the Effects of Nonuniform Spreading Velocity of Subma- rine Slumps and Slides on the Near-Field Tsunami Ampli- tudes," Soil Dynamics and Earthquake Engineering, Vol. 22, No. 3, 2002, pp. 167-180.

[25] M. D. Trifunaca, A. Hayira and M. I. Todorovska, "Was Grand Banks Event of 1929 a Slump Spreading in Two Directions," Soil Dynamics and Earthquake Engineering, Vol. 22, No. 5, 2002, pp. 349-360.

[26] J. L. Hammack, "A Note on Tsunamis: Their Generation and Propagation in an Ocean of Uniform Depth," Journal of Fluid Mechanics, Vol. 60, No. 4, 1973, pp. 769-799.

[27] D. Dutykh and F. Dias, "Water Waves Generated by a Moving Bottom,” In K. Anjan, Ed. Tsunami and Nonlinear waves, Springer-Verlag, Berlin, 2007, pp. 63-94.
[28] N. A. Haskell, "Elastic Displacements in the Near-Field of a Propagating Fault," Bulletin of the Seismological Society of America, Vol. 59, No. 2, 1969, pp. 865-908.

[29] V. V. Titov and F. I. Gonzalez, "Implementation and Testing of the Method of Splitting Tsunami (MOST) Model," NOAA/Pacific Marine Environmental Laboratory, No. 1927, 1997.

[30] A. Y. Bezhaev, M. M. Lavrentiev, A. G. Marchuk and V. V. Titov, "Determination of Tsunami Sources Using Deep Ocean Wave Records," Center Mathematical Models in Geophysics, Bull. Nov. Comp., Vol. 11, 2006, pp. 53-63.

[31] D. R. Fuhrman and P. A. Madsen, "Tsunami Generation, Propagation, and Run-up with a High-Order Boussinesq Model," Coastal Engineering, Vol. 56, No. 7, 2009, pp. 747-758.

[32] X. Zhao, B. Wang and H. Liu, "Modeling the Submarine Mass Failure Induced Tsunamis by Boussinesq Equations," Journal of Asian Earth Sciences, Vol. 36, No. 4, 2009, pp. 47-55.

[33] H. Benioff and F. Pess, "Progress Report on Long Period Seismographs," Geophysical Journal International, Vol. 1, No. 3, 1958, pp. 208-215.

[34] H. Kanamori and G. S. Stewart, "A Slowly Earthquake," Physics of the Earth and Planetary Interiors, Vol. 18, No. 3, 1972, pp. 167-175.

[35] P. G. Silver and T. H. Jordan, "Total-Moment Spectra of Fourteen Large Earthquakes," Journal of Geophysical Research, Vol. 88, No. B4, 1983, pp. 3273-3293.

[36] A. Hayir, "Ocean Depth Effects on Tsunami Amplitudes Used in Source Models in Linearized Shallow-Water Wave Theory," Ocean Engineering, Vol. 31, No. 3-4, 2004, pp. 353-361. 


\section{APPENDIX I}

The equations for conservation of mass and momentum for an inviscid, incompressible fluid are

$$
\begin{array}{cc}
\text { Mass: } & \nabla \cdot \mathbf{u}=0 \\
\text { Momentum: } & \frac{\partial \mathbf{u}}{\partial \mathrm{t} \rho}+\mathbf{u} . \nabla \mathbf{u}=-\nabla\left(\frac{\mathbf{P}}{+\mathrm{gz}}\right)
\end{array}
$$

where $\mathbf{u}(\mathbf{x}, \mathrm{t})$ is the velocity vector $(\mathrm{u}, \mathrm{v}, \mathrm{w})$ of the fluid, $\mathbf{P}(\mathbf{x}, \mathrm{t})$ is the pressure vector, $\rho$ the density, $\mathrm{g}$ the gravitational acceleration, and $\mathbf{x}=(\mathrm{x}, \mathrm{y}, \mathrm{z})$ with the $z$ axis pointing vertically upward.

By assuming that the flow is irrotational, hence the velocity field $\mathbf{u}$ can be written as the gradiant of the potential function $\mathbf{u}=\nabla \boldsymbol{\phi}$, where $\boldsymbol{\phi}$ is the velocity potential. Then the continuity equation becomes the Laplace's equation

$$
\nabla^{2} \phi=0 .
$$

Mathematically, a general solution does not exist for gravity waves and approximations must be made for even simple waves. One of the important problems in water wave theory is to establish the limits of validity of the various solutions that are due to the simplifying assumptions. The mathematical treatments of water wave motion use all the mathematical physics dealing with linear and nonlinear problems. The main difficulty in the study of water motion is that the free surface boundary is unknown. The coordinate axis that will be used to describe wave motion will be located at the vertical displacement $\mathrm{z}$ ( $(x, y, t)$. The bottom of the water body will be at $\mathrm{z}$ 兴( $(\mathrm{xh} t \mathrm{t})$

If the velocity potential is known, then the pressure field can be found from (A.2). By using the vector identity

$$
\text { u. } \nabla \mathbf{u}=\nabla \frac{\mathrm{u}^{2}}{2}-\mathbf{u} \times(\nabla \times \mathbf{u})
$$

From irrotationality (i.e. $\nabla \times \mathbf{u}=0$ ), (A.2) may be rewritten as

$$
\nabla\left[\frac{\partial \phi}{\partial \mathrm{t}}+\frac{1}{\rho}|\nabla \phi|^{2}\right]=-\nabla\left(\frac{\mathbf{P}}{\mathrm{gz}}\right) .
$$

Upon integration with respect to the space variables, we obtain

$$
-\frac{\mathrm{P}}{\rho \mathrm{t}}=\mathrm{gz}+\frac{\partial \phi}{\partial}+\frac{1}{2}|\nabla \phi|^{2}+\mathrm{C}(\mathrm{t}),
$$

where $\mathrm{C}(\mathrm{t})$ is an arbitrary function of $t$ and can usually be omitted by redefining $\phi$ without affecting the velocity field. Equation (A.6) is called the Bernoulli equation. The first term gz, on the right-hand side of (A.6) is the hydrostatic contribution, whereas the hydrodynamic contribution to the total pressure $\mathrm{P}$.
Two types of boundaries interest us: the air-water interface which will also be called the free surface and the wetted surface of an impenetrable solid (bottom surface). Along these two boundaries the fluid is assumed to move only tangentially. Let the instantaneous equation of the boundary be

$$
\mathrm{F}(\mathrm{x}, \mathrm{t})=\mathrm{z}-\eta(\mathrm{x}, \mathrm{y}, \mathrm{t})=0
$$

where $\eta$ is the height measured from $\mathrm{z}=0$, and let the velocity of a geometrical point $x$ of the moving free surface be $q$.

After short time $\mathrm{dt}$, the free surface is described by

$$
\begin{aligned}
& \mathrm{F}(\mathrm{x}+\mathrm{qdt}, \mathrm{t}+\mathrm{dt})=0= \\
& \mathrm{F}(\mathrm{x}, \mathrm{t})+\left(\frac{\partial \mathrm{F}}{\partial \mathrm{t}}+\mathrm{q} \nabla \mathrm{F}\right) \mathrm{dt}+\mathrm{O}(\mathrm{dt})^{2}
\end{aligned}
$$

In view of (A.7), it follows that

$$
\frac{\partial \mathrm{F}}{\partial \mathrm{t}}+\mathrm{q} \cdot \nabla \mathrm{F}=0 \text {. }
$$

For small but arbitrary $\mathrm{dt}$.The assumption of tangential motion requires $\mathrm{u} . \nabla \mathrm{F}=\mathrm{q} . \nabla \mathrm{F}$.

This in turn implies that

$$
\frac{\partial \mathrm{F}}{\partial \mathrm{t}}+\mathrm{u} . \nabla \mathrm{F}=0 \quad \text { on } \quad \mathrm{z} f(\mathrm{x}, \mathrm{y}, \mathrm{t})
$$

or equivalently

$$
\frac{\partial \eta \eta \eta}{\partial \mathrm{t}}+\frac{\partial \phi}{\partial \mathrm{x}} \frac{\partial}{\partial \mathrm{x}}+\frac{\partial \phi}{\partial \mathrm{y}} \frac{\partial}{\partial \mathrm{y}}=\frac{\partial \phi}{\partial \mathrm{z}} \quad \text { on } \quad \mathrm{z} \neq(\mathrm{x}, \mathrm{y}, \mathrm{t})
$$

On the sea bottom $\zeta(\mathrm{x}, \mathrm{y}, \mathrm{t})$ at depth $\mathrm{h},(\mathrm{A} .7)$ becomes $\mathrm{z}+\mathrm{h}-\zeta(\mathrm{x}, \mathrm{y}, \mathrm{t})=0$ and (A.9) may be written by the same way as:

$$
\frac{\partial \zeta \zeta \zeta}{\partial \mathrm{t}}+\frac{\partial \phi}{\partial \mathrm{x}} \frac{\partial}{\partial \mathrm{x}}+\frac{\partial \phi}{\partial \mathrm{y}} \frac{\partial}{\partial \mathrm{y}}=\frac{\partial \phi}{\partial \mathrm{z}} \quad \text { on } \quad \mathrm{z} \xi(\mathrm{xh}, \mathrm{y}, \mathrm{t})
$$

Equations (A.11) and (A.12) are referred to the kinematic boundary conditions.

On the air-water interface, both $\eta$ and $\phi$ are unknown and it is necessary to add a dynamic boundary condition concerning forces.

The wavelength is so long that surface tension is unimportant, and hence the pressure at the beneath the free surface must equal to the atmospheric pressure $\mathrm{P}_{\mathrm{a}}$ above. It can be taken $\mathrm{P}=0$ using the simple transformation $\mathrm{P} \rightarrow \mathrm{P}-\mathrm{P}_{\mathrm{a}}$ which does not change the basic Euler equations which depend upon $\nabla \mathrm{P}$. Hence Bernoulli Equation (A.6) at the free surface gives the boundary condition

$$
\frac{\partial \phi}{\partial \mathrm{t}}+\frac{1}{2}|\nabla \phi|^{2}+\mathrm{g \eta} \theta \quad \text { on } \quad \mathrm{z}=\eta(\mathrm{x}, \mathrm{y}, \mathrm{t}) .
$$

This is known as the dynamic boundary condition at the free surface. 